\title{
28. DATA REPORT: GEOCHEMISTRY OF ROCKS AND MINERALS OF THE GABBRO COMPLEX FROM THE MARK AREA ${ }^{1}$
}

\author{
Carl-Dietrich Werner ${ }^{2}$
}

\section{INTRODUCTION}

Leg 153 represents an Ocean Drilling Program (ODP) offset drilling leg in the MARK area (Mid-Atlantic Ridge at the Kane Transform), around $22^{\circ} 33^{\prime} \mathrm{N}, 45^{\circ} 1.9^{\prime} \mathrm{W}$. Shipboard documentation has shown that the gabbroic rocks from Sites 921,922 , and 923 are part of a layered complex and are generally similar, although cumulus textures are particularly well developed in the rocks from Site 922. These rocks also exhibit some special geochemical features.

Major- and trace-element chemistries were studied from a limited pool of 26 samples from nine holes. In some polished thin sections, the chemical compositions of olivines, orthopyroxenes, clinopyroxenes, amphiboles, and plagioclases were determined using an electron microprobe. These data are presented here as a data report; a detailed paper will be prepared, if additional rock samples are analyzed, and if fluid inclusions and oxygen isotope data become available.

\section{SAMPLE MATERIAL}

All gabbroic rock samples in this study are from Holes 153-921A, 921B, 921C, 921E, 922A, 922B, and 923A. Two diabase vein rocks are from Holes 920B and 920D. The special designations correspond to shipboard documentation and to standard ODP sample nomenclature conventions, but, for clarity, single numbers are generally used in the data tables (Table 1). The individual rock names were determined after microscopic investigation and calculation of mineral norms in accordance with the CIPW system (after the authors Cross, Iddings, Pearson, and Washington).

\section{ANALYTICAL METHODS Whole-Rock Chemistry}

Major-element oxides and the trace elements $\mathrm{Cl}, \mathrm{Co}, \mathrm{Cr}, \mathrm{Cu}, \mathrm{F}$, $\mathrm{Ga}, \mathrm{Nb}, \mathrm{Ni}, \mathrm{Pb}, \mathrm{S}, \mathrm{Sc}, \mathrm{V}, \mathrm{Y}, \mathrm{Zn}$, and $\mathrm{Zr}$ were measured by $\mathrm{X}$-ray fluorescence spectroscopy using a Phillips PW 1480 sequential spectrometer. Glass discs were prepared by melting the powdered, but not ignited, whole-rock material with lithium tetraborate. International reference rocks, as well as two internal mafic standard samples, were used for calibration of the method.

$\mathrm{K}, \mathrm{Li}, \mathrm{Na}, \mathrm{Rb}$, and $\mathrm{Zn}$ were analyzed using flame photometry, and $\mathrm{Ba}$ and $\mathrm{Sr}$ were analyzed using inductively coupled plasma-atomic emission spectroscopy using a Perkin-Elmer PLASMA 1000 spectrometer. B was determined by optical spectroscopy, with boron-free spectral carbons, using a Carl Zeiss PGS 2 spectrograph.

'Karson, J.A., Cannat, M., Miller, D.J., and Elthon, D. (Eds.), 1997. Proc. ODP, Sci. Results, 153: College Station, TX (Ocean Drilling Program).

${ }^{2} \mathrm{TU}$ Bergakademie Freiberg, Institut für Mineralogie, D-09596 Freiberg, Federal Republic of Germany.
Table 1. Sample information.

\begin{tabular}{|c|c|c|c|}
\hline Sample name & Hole, core, section, piece & $\begin{array}{l}\text { Depth } \\
\text { (mbsf) }\end{array}$ & Rock name \\
\hline $2 \mathrm{D}$ & 920B-9R-3 (Piece 12A) & 83.7 & Diabase \\
\hline $7 \mathrm{M}-\mathrm{D}$ & $920 \mathrm{D}-9 \mathrm{R}-2$ (Piece 6B) & 78.1 & Metadiabase \\
\hline $100 \mathrm{GbNo}$ & $921 \mathrm{~A}-2 \mathrm{R}-1$ (Piece 7) & 8.3 & Gabbronorite \\
\hline $102 \mathrm{~Gb}$ & $921 \mathrm{~B}-1 \mathrm{~W}-2$ (Piece 3 ) & 1.6 & Gabbro \\
\hline $103 \mathrm{GbNo}$ & $921 \mathrm{~B}-2 \mathrm{R}-2$ (Piece 7 & 16.9 & Gabbronorite \\
\hline $104 \mathrm{OlGb}$ & 921B-4R-1 (Piece 4) & 34.4 & Olivine gabbro \\
\hline $142 \mathrm{OlGN}$ & $921 \mathrm{~B}-4 \mathrm{R}-3$ (Piece 3 ) & 37.2 & Olivine gabbronorite \\
\hline $108 \mathrm{OlGb}$ & 921 C-3R-1 (Piece 5A) & 20.1 & Olivine gabbro \\
\hline $109 \mathrm{OlGb}$ & $921 \mathrm{C}-3 \mathrm{R}-3$ (Piece 3 ) & 22.7 & Olivine gabbro \\
\hline $110 \mathrm{~Gb}$ & $921 \mathrm{E}-2 \mathrm{R}-1$ (Piece 5B) & 10.6 & Gabbro \\
\hline $113 \mathrm{GbNo}$ & $921 \mathrm{E}-4 \mathrm{R}-2$ (Piece 1B) & 31.2 & Gabbronorite \\
\hline $114 \mathrm{OlGb}$ & 921E-5R-1 (Piece 5) & 40.0 & Olivine gabbro \\
\hline $115 \mathrm{OlGN}$ & 921E-5R-1 (Piece 11) & 40.5 & Olivine gabbronorite \\
\hline $116 \mathrm{OlGb}$ & 921 E-5R-2 (Piece 6) & 41.8 & Olivine gabbro \\
\hline $118 \mathrm{~Gb}$ & 921 E-6R-1 (Piece 12) & 50.8 & Gabbro \\
\hline 119 OIGb & 921 E-7R-3 (Piece 2) & 63.1 & Olivine gabbro \\
\hline $121 \mathrm{TR}$ & $922 \mathrm{~A}-2 \mathrm{R}-1$ (Piece 8 ) & 7.7 & Troctolite \\
\hline $122 \mathrm{TR}$ & $922 \mathrm{~A}-2 \mathrm{R}-2$ (Piece 1C) & 8.6 & Troctolite \\
\hline 123 OINo & 922A-2R-4 (Piece 2A) & 11.3 & Olivine norite \\
\hline 124 OINo & $922 \mathrm{~A}-2 \mathrm{R}-5$ (Piece 2B) & 13.2 & Olivine norite \\
\hline $125 \mathrm{OlGb}$ & $922 \mathrm{~A}-3 \mathrm{R}-1$ (Piece 4A) & 14.2 & Olivine gabbro \\
\hline $126 \mathrm{OIGN}$ & $922 \mathrm{~B}-1 \mathrm{~W}-1$ (Piece 7) & 1.0 & Olivine gabbronorite \\
\hline 127 OlNo & $922 \mathrm{~B}-2 \mathrm{R}-1$ (Piece 1C) & 14.6 & Olivine norite \\
\hline 129 OIGN & 922B-3R-1 (Piece 4A) & 19.8 & Olivine gabbronorite \\
\hline $130 \mathrm{OINo}$ & $922 \mathrm{~B}-3 \mathrm{R}-2$ (Piece 1) & 20.3 & Olivine norite \\
\hline $133 \mathrm{GbNo}$ & $923 \mathrm{~A}-2 \mathrm{R}-2$ (Piece 1) & 17.7 & Gabbronorite \\
\hline $134 \mathrm{OlGb}$ & 923A-7R-1 (Piece 2B) & 27.0 & Olivine gabbro \\
\hline $136 \mathrm{GbNo}$ & $923 \mathrm{~A}-10 \mathrm{R}-2$ (Piece $2 \mathrm{~A})$ & 38.5 & Gabbronorite \\
\hline $137 \mathrm{~Gb}$ & $923 \mathrm{~A}-10 \mathrm{R}-3$ (Piece $2 \mathrm{~A}$ ) & 39.4 & Gabbro \\
\hline $138 \mathrm{~Gb}$ & $923 \mathrm{~A}-11 \mathrm{R}-2($ Piece $1 \mathrm{~A})$ & 42.6 & Gabbro \\
\hline 139 OlGN & 923 A-15R-3 (Piece1) & 63.0 & Olivine gabbronorite \\
\hline $141 \mathrm{GbNo}$ & $923 \mathrm{~A}-16 \mathrm{R}-4$ (Piece 6) & 68.9 & Gabbronorite \\
\hline GABI OlGb* & & & Olivine gabbro \\
\hline
\end{tabular}

Note: * = Leg 153 internal laboratory standard.

The results of the chemical investigation are compiled in Table 2 (major-element oxides in wt\%) and Table 3 (trace elements in ppm). The CIPW norm mineral data are presented in Table 4 and supplemented by the Thornton-Tuttle Index (TTI: $Q+$ or $+a b+l c+n e$ ), normative anorthite content in plagioclase $\left(\mathrm{An}^{*}\right)$, and the $\mathrm{Mg}$ number $(\mathrm{Mg} \#=\mathrm{Mg} /[\mathrm{Mg}+\mathrm{Fe}])$. Selected element ratios are also listed in $\mathrm{Ta}-$ ble 4 .

Finally, Table 5 presents the mean values of our analytical data from Leg 153 gabbroic rock types (major-element oxides and trace elements); results of calculations of the CIPW norms are listed in Table 6 . Initial views of the positions of the individual gabbroic rocks are indicated in Figures 1-5.

\section{Mineral Chemistry}

The chemical composition of the individual minerals was measured from polished thin sections on a WDX microprobe, using the CAMEBAX S 50 at the Ruhr-Universität Bochum. Several synthetic reference samples were available for calibration.

Altogether, more than 2500 points were analyzed, hence, only a selection of typical data is given in Tables 7-11. In most cases, the 
Table 2. Major-element compositions (wt \%) of Leg 153 gabbros.

\begin{tabular}{|c|c|c|c|c|c|c|c|c|c|c|c|}
\hline Sample no.: & 100 & 103 & 104 & 142 & 108 & 109 & 113 & 115 & 118 & 119 & 121 \\
\hline Rock name: & GbNo & GbNo & OIGb & OIGN & OlGb & $\mathrm{OlGb}$ & GbNo & OlGN & $\mathrm{Gb}$ & $\mathrm{OlGb}$ & TR \\
\hline Hole: & $921 \mathrm{~A}$ & $921 \mathrm{~B}$ & $921 \mathrm{~B}$ & $921 \mathrm{~B}$ & $921 \mathrm{C}$ & $921 \mathrm{C}$ & $921 \mathrm{E}$ & $921 \mathrm{E}$ & $921 \mathrm{E}$ & $921 \mathrm{E}$ & $922 \mathrm{~A}$ \\
\hline Core: & $2 \mathrm{R}$ & $2 \mathrm{R}$ & $4 \mathrm{R}$ & $4 \mathrm{R}$ & $3 R$ & $3 R$ & $4 \mathrm{R}$ & $5 R$ & $6 \mathrm{R}$ & $7 \mathrm{R}$ & $2 \mathrm{R}$ \\
\hline Section, piece: & 1,7 & 2,7 & 1,4 & 3,3 & $1,5 \mathrm{~A}$ & 3,3 & $2,1 B$ & 1,11 & 1,12 & 3,2 & 1,8 \\
\hline Depth (mbsf): & 8.3 & 16.9 & 34.4 & 37.2 & 20.1 & 22.7 & 31.2 & 40.5 & 50.8 & 63.1 & 7.7 \\
\hline $\mathrm{SiO}_{2}$ & 52.7 & 50.9 & 49.1 & 47.1 & 48.8 & 48.6 & 50.4 & 49.5 & 50.7 & 49.5 & 45.2 \\
\hline $\mathrm{TiO}_{2}^{2}$ & 0.43 & 0.47 & 0.38 & 0.19 & 0.32 & 0.38 & 0.41 & 0.22 & 0.4 & 0.28 & 0.19 \\
\hline $\mathrm{Al}_{2} \mathrm{O}_{3}$ & 16.3 & 16.5 & 17.1 & 19.1 & 18.3 & 17.3 & 15.8 & 19.5 & 16.6 & 18.4 & 18.5 \\
\hline $\mathrm{Cr}_{2} \mathrm{O}_{3}$ & 0.011 & 0.026 & 0.14 & 0.033 & 0.29 & 0.27 & 0.056 & 0.076 & 0.052 & 0.078 & 0.050 \\
\hline $\mathrm{Fe}_{2} \mathrm{O}_{3}$ & 7.18 & 6.1 & 6.95 & 7.5 & 4.35 & 4.8 & 7.22 & 4.7 & 5.26 & 5.14 & 6.42 \\
\hline $\mathrm{MnO}$ & 0.157 & 0.111 & 0.124 & 0.116 & 0.077 & 0.089 & 0.136 & 0.082 & 0.109 & 0.092 & 0.107 \\
\hline $\mathrm{MgO}$ & 8.15 & 9.1 & 10.3 & 11.9 & 10.6 & 11.4 & 9.9 & 10.0 & 9.1 & 9.8 & 16.6 \\
\hline $\mathrm{NiO}$ & 0.009 & 0.014 & 0.025 & 0.038 & 0.033 & 0.035 & 0.017 & 0.027 & 0.016 & 0.027 & 0.072 \\
\hline $\mathrm{CaO}$ & 10.8 & 12.7 & 12.5 & 10.2 & 14.2 & 13.8 & 12.7 & 12.3 & 14.3 & 13.4 & 9.8 \\
\hline $\mathrm{Na}_{2} \mathrm{O}$ & 3.25 & 2.65 & 2.3 & 2.2 & 1.85 & 1.85 & 2.25 & 2.15 & 2.3 & 2.2 & 1.45 \\
\hline $\mathrm{K}_{2} \mathrm{O}$ & 0.044 & 0.046 & 0.041 & 0.046 & 0.024 & 0.032 & 0.029 & 0.028 & 0.039 & 0.033 & 0.061 \\
\hline $\mathrm{P}_{2} \mathrm{O}_{5}$ & 0.018 & 0.085 & 0.026 & 0.021 & 0.02 & 0.029 & 0.024 & 0.019 & 0.020 & 0.017 & 0.035 \\
\hline LOI & 0.85 & 1.1 & 0.8 & 1.7 & 1.0 & 1.25 & 0.7 & 1.2 & 0.8 & 0.85 & 1.5 \\
\hline Total & 99.90 & 99.80 & 99.79 & 100.14 & 99.86 & 99.82 & 99.64 & 99.80 & 99.70 & 99.82 & 99.98 \\
\hline
\end{tabular}

Table 2 (continued).

\begin{tabular}{|c|c|c|c|c|c|c|c|c|c|c|}
\hline Sample no.: & 122 & 123 & 124 & 126 & 127 & 129 & 130 & 133 & 134 & 136 \\
\hline Rock name: & TR & OlNo & OlNo & OlGN & OINo & OIGN & OlNo & GbNo & $\mathrm{OlGb}$ & GbNo \\
\hline Hole: & $922 \mathrm{~A}$ & $922 \mathrm{~A}$ & $922 \mathrm{~A}$ & $922 \mathrm{~B}$ & $922 \mathrm{~B}$ & $922 \mathrm{~B}$ & $922 \mathrm{~B}$ & $923 \mathrm{~A}$ & $923 \mathrm{~A}$ & $923 \mathrm{~A}$ \\
\hline Core: & $2 \mathrm{R}$ & $2 \mathrm{R}$ & $2 \mathrm{R}$ & IW & $2 \mathrm{R}$ & $3 R$ & $3 R$ & $2 \mathrm{R}$ & $7 \mathrm{R}$ & $10 \mathrm{R}$ \\
\hline Section, piece: & $2,1 \mathrm{C}$ & $4,2 \mathrm{~A}$ & $5,2 \mathrm{~B}$ & 1,7 & $1,1 \mathrm{C}$ & $1,4 \mathrm{~A}$ & 2,1 & 2,1 & $1,2 \mathrm{~B}$ & $2,2 \mathrm{~A}$ \\
\hline Depth (mbsf): & 8.6 & 11.3 & 13.2 & 1.0 & 14.6 & 19.8 & 20.3 & 17.7 & 27.0 & 38.5 \\
\hline $\mathrm{SiO}_{2}$ & 46.5 & 46.7 & 47.6 & 46.9 & 46.4 & 47.3 & 45.2 & 51.9 & 49.8 & 50.6 \\
\hline $\mathrm{TiO}_{2}$ & 0.17 & 0.65 & 0.75 & 0.63 & 0.28 & 0.38 & 0.7 & 0.39 & 0.30 & 0.33 \\
\hline $\mathrm{Al}_{2} \mathrm{O}_{3}$ & 22.2 & 17.1 & 21.85 & 18.3 & 20.3 & 18.8 & 17.9 & 15.9 & 18.6 & 17.4 \\
\hline $\mathrm{Cr}_{2} \mathrm{O}_{3}$ & 0.012 & 0.014 & 0.008 & 0.145 & 0.045 & 0.065 & 0.010 & 0.018 & 0.018 & 0.118 \\
\hline $\mathrm{Fe}_{2} \mathrm{O}_{3}$ & 5.28 & 10.3 & 6.71 & 7.25 & 5.7 & 5.72 & 7.86 & 7.38 & 6.72 & 6.56 \\
\hline $\mathrm{MnO}$ & 0.084 & 0.183 & 0.108 & 0.12 & 0.080 & 0.085 & 0.107 & 0.15 & 0.115 & 0.118 \\
\hline $\mathrm{MgO}$ & 11.9 & 12.0 & 7.2 & 10.9 & 11.5 & 11.8 & 14.5 & 8.6 & 9.1 & 8.7 \\
\hline $\mathrm{NiO}$ & 0.051 & 0.044 & 0.024 & 0.043 & 0.049 & 0.040 & 0.057 & 0.010 & 0.018 & 0.015 \\
\hline $\mathrm{CaO}$ & 11.05 & 9.45 & 11.1 & 11.5 & 10.8 & 11.15 & 9.0 & 11.7 & 11.9 & 12.6 \\
\hline $\mathrm{Na}_{2} \mathrm{O}$ & 1.9 & 1.75 & 2.6 & 1.93 & 1.85 & 1.9 & 1.51 & 3.0 & 2.65 & 2.6 \\
\hline $\mathrm{K}_{2} \mathrm{O}$ & 0.057 & 0.058 & 0.061 & 0.063 & 0.049 & 0.055 & 0.08 & 0.062 & 0.048 & 0.035 \\
\hline $\mathrm{P}_{2} \mathrm{O}_{5}$ & 0.044 & 0.152 & 0.33 & 0.18 & 0.040 & 0.048 & 0.051 & 0.029 & 0.022 & 0.017 \\
\hline LOI & 1.2 & 1.6 & 1.5 & 2.2 & 2.8 & 2.7 & 2.9 & 0.55 & 0.5 & 0.7 \\
\hline Total & 100.35 & 100.00 & 99.84 & 100.16 & 99.89 & 100.04 & 99.88 & 99.69 & 99.79 & 99.79 \\
\hline
\end{tabular}

Table 2 (continued).

\begin{tabular}{|c|c|c|c|c|c|c|c|}
\hline Sample no.: & 137 & 138 & 139 & 141 & 2 & 7 & \\
\hline Rock name: & $\mathrm{Gb}$ & $\mathrm{Gb}$ & OlGN & GbNo & D & $M-D$ & \\
\hline Hole: & $923 \mathrm{~A}$ & $923 \mathrm{~A}$ & $923 \mathrm{~A}$ & $923 \mathrm{~A}$ & $920 \mathrm{~B}$ & 920D & \\
\hline Core: & IOR & $11 \mathrm{R}$ & $15 \mathrm{R}$ & $16 \mathrm{R}$ & $9 \mathrm{R}$ & $9 \mathrm{R}$ & \\
\hline Section, piece: & $3,2 \mathrm{~A}$ & $2,1 \mathrm{~A}$ & 3,1 & 4,6 & $3,12 \mathrm{~A}$ & $2,6 \mathrm{~B}$ & GABI \\
\hline Depth (mbsf): & 39.4 & 42.6 & 63.0 & 68.9 & 83.7 & 78.1 & OlGb \\
\hline $\mathrm{SiO}_{2}$ & 50.6 & 50.4 & 47.9 & 50.8 & 46.4 & 46.2 & 48.0 \\
\hline $\mathrm{TiO}_{2}$ & 0.42 & 0.39 & 0.17 & 0.48 & 0.96 & 0.92 & 0.26 \\
\hline $\mathrm{Al}_{2} \mathrm{O}_{3}$ & 16.1 & 15.2 & 21.9 & 15.7 & 16.3 & 15.7 & 17.8 \\
\hline $\mathrm{Cr}_{2} \mathrm{O}_{3}$ & 0.031 & 0.051 & 0.065 & 0.077 & 0.051 & 0.063 & 0.26 \\
\hline $\mathrm{Fe}_{2} \mathrm{O}_{3}$ & 6.91 & 6.75 & 5.05 & 6.01 & 8.51 & 8.85 & 4.54 \\
\hline $\mathrm{MnO}$ & 0.13 & 0.127 & 0.077 & 0.122 & 0.145 & 0.24 & 0.085 \\
\hline $\mathrm{MgO}$ & 9.1 & 9.8 & 8.8 & 9.2 & 11.7 & 13.4 & 11.4 \\
\hline $\mathrm{NiO}$ & 0.013 & 0.016 & 0.028 & 0.013 & 0.029 & 0.036 & 0.034 \\
\hline $\mathrm{CaO}$ & 13.2 & 13.4 & 12.0 & 13.6 & 9.9 & 7.4 & 14.2 \\
\hline $\mathrm{Na}_{2} \mathrm{O}$ & 2.7 & 2.45 & 2.35 & 2.3 & 2.3 & 2.9 & 1.75 \\
\hline $\mathrm{K}_{2} \mathrm{O}$ & 0.038 & 0.036 & 0.033 & 0.044 & 0.042 & 0.054 & 0.028 \\
\hline $\mathrm{P}_{2} \mathrm{O}_{5}$ & 0.021 & 0.019 & 0.019 & 0.041 & 0.08 & 0.08 & 0.015 \\
\hline $\mathrm{LOI}$ & 0.5 & 1.3 & 1.5 & 1.4 & 3.5 & 4.1 & 1.5 \\
\hline Total & 99.76 & 99.94 & 99.89 & 99.79 & 99.92 & 99.94 & 99.87 \\
\hline
\end{tabular}

Notes: $\mathrm{GbNo}=$ gabbronorite, $\mathrm{OlGb}=$ olivine gabbro, $\mathrm{OlGN}=$ olivine gabbronorite, $\mathrm{Gb}=$ gabbro, $\mathrm{TR}=$ troctolite, $\mathrm{OlNo}=$ olivine norite, $\mathrm{D}=$ diabase, $\mathrm{M}-\mathrm{D}=$ metadiabase. $\mathrm{LOI}=$ loss on ignition. 
Table 3. Trace-element compositions (ppm) of Leg 153 gabbros.

\begin{tabular}{|c|c|c|c|c|c|c|c|c|c|c|c|}
\hline $\begin{array}{l}\text { Sample no.: } \\
\text { Rock name: }\end{array}$ & $\begin{array}{c}100 \\
\text { GbNo }\end{array}$ & $\begin{array}{c}103 \\
G b N o\end{array}$ & $\begin{array}{c}104 \\
\text { OlGb }\end{array}$ & 142 & 108 & $\begin{array}{c}109 \\
01 G b\end{array}$ & 113 & 115 & 118 & 119 & $\begin{array}{l}121 \\
T R\end{array}$ \\
\hline & & & & & & & & & & & \\
\hline B & 5 & 6 & 5 & 6 & 4 & 5 & 6 & 4 & 5 & 5 & 4 \\
\hline $\mathrm{Li}$ & 5 & 6 & 8 & 7 & 7 & 9 & 8 & 9 & 9 & 8 & 8 \\
\hline $\mathrm{Rb}$ & - & 1 & 2 & $\sim 1$ & - & $<1$ & - & - & 1 & 2 & $<1$ \\
\hline $\mathrm{Sr}$ & 150 & 134 & 125 & 148 & 121 & 112 & 125 & 133 & 138 & 142 & 126 \\
\hline $\mathrm{Ba}$ & 11 & 6.7 & 8.6 & 18 & 18 & 8.5 & 8.4 & 12 & 9 & 6.1 & 12.3 \\
\hline $\mathrm{Ga}$ & 15 & 14 & 9 & 10 & 10 & 11 & 10 & 9 & 12 & 10 & \\
\hline $\mathrm{Pb}$ & & 6 & 6 & 8 & 3 & - & 2 & 4 & 3 & 4 & 4 \\
\hline $\mathrm{Cu}$ & 33 & 78 & 94 & 72 & 88 & 101 & 82 & 79 & 65 & 93 & 64 \\
\hline $\mathrm{Zn}$ & 48 & 39 & 45 & 50 & 24 & 30 & 41 & 29 & 32 & 28 & 52 \\
\hline $\mathrm{Y}$ & 8 & 13 & 8 & 4 & 9 & 12 & 10 & 5 & 8 & 6 & 9 \\
\hline $\mathrm{Nb}$ & - & $\sim 2$ & - & -1 & $<1$ & $\sim 1$ & $\sim 1$ & $<1$ & $\sim 1$ & $<1$ & $\sim 1$ \\
\hline $\mathrm{Zr}$ & 16 & 45 & 19 & 14 & 14 & 23 & 20 & 13 & 18 & 14 & 21 \\
\hline V & 180 & 162 & 129 & 53 & 127 & 137 & 168 & 88 & 186 & 110 & 42 \\
\hline $\mathrm{Ni}$ & 70 & 108 & 195 & 300 & 260 & 275 & 135 & 215 & 125 & 210 & 565 \\
\hline Co & 36 & 38 & 40 & 52 & 35 & 33 & 34 & 36 & 28 & 39 & 47 \\
\hline $\mathrm{Cr}$ & 74 & 180 & 965 & 225 & 1965 & 1855 & 385 & 520 & 355 & 535 & 340 \\
\hline $\mathrm{Sc}$ & 37 & $\begin{array}{r}420 \\
\end{array}$ & 29 & 14 & 35 & 34 & 49 & 20 & 50 & 33 & 9 \\
\hline s & 205 & 300 & 425 & 400 & 330 & 455 & 585 & 335 & 265 & 305 & 260 \\
\hline As & 3.6 & 3.1 & 1.5 & 9.3 & 2.5 & 3.1 & 15 & 5.3 & 2.5 & 17 & 42 \\
\hline $\mathrm{Sb}$ & 0.03 & 0.02 & & 0.05 & 0.15 & - & 0.12 & 1.06 & 0.11 & 6.5 & 0.37 \\
\hline $\mathrm{Cl}$ & $<21$ & $<45$ & 1135 & $<52$ & $<40$ & $<88$ & 1100 & $<25$ & $<51$ & $<24$ & 195 \\
\hline $\mathrm{F}$ & $<86$ & 330 & 190 & - & 310 & 355 & 265 & 350 & 390 & 220 & 205 \\
\hline
\end{tabular}

Table 3 (continued).

\begin{tabular}{|c|c|c|c|c|c|c|c|c|c|c|}
\hline Sample no.: & 122 & 123 & 124 & 126 & 127 & 129 & 130 & 133 & 134 & 136 \\
\hline Rock name: & TR & OlNo & OINo & OlGN & OlNo & OlGN & OINo & GbNo & $\mathrm{OlGb}$ & GbNo \\
\hline B & 4 & 3 & 3 & 4 & 4 & 4 & 5 & 3 & 4 & 5 \\
\hline $\mathrm{Li}$ & 8 & 4 & 5 & 6 & 6 & 5 & 5 & 6 & 8 & 7 \\
\hline $\mathrm{Rb}$ & 2 & - & $\sim 1$ & -2 & $\sim 2$ & $\sim 2$ & 1 & 2 & $\sim 1$ & $\sim 1$ \\
\hline $\mathrm{Sr}$ & 152 & 129 & 166 & 128 & 129 & 132 & 117 & 145 & 152 & 149 \\
\hline $\mathrm{Ba}$ & 10 & 15 & 10.5 & 7.6 & 9.8 & 10.1 & 10 & 10.3 & 8.7 & 11.8 \\
\hline $\mathrm{Ga}$ & 12 & 8 & 15 & 12 & 11 & 12 & 9 & 12 & 15 & 12 \\
\hline $\mathrm{Pb}$ & 5 & 3 & 4 & 6 & 3 & 3 & $<1$ & 6 & $<2$ & $<2$ \\
\hline $\mathrm{Cu}$ & 38 & 88 & 36 & 68 & 25 & 23 & 36 & 71 & 60 & 79 \\
\hline $\mathrm{Zn}$ & 43 & 96 & 56 & 66 & 46 & 51 & 64 & 45 & 41 & 48 \\
\hline $\mathrm{Y}$ & 10 & 26 & 30 & 28 & 16 & 18 & 12 & 16 & 8 & 9 \\
\hline $\mathrm{Nb}$ & 2 & 3 & 5 & 5 & 3 & 4 & 3 & 3 & $\sim 1$ & $<1$ \\
\hline $\mathrm{Zr}$ & 32 & 58 & 98 & 82 & 66 & 78 & 48 & 72 & 20 & 9 \\
\hline V & 27 & 76 & 105 & 120 & 53 & 90 & 80 & 164 & 108 & 130 \\
\hline $\mathrm{Ni}$ & 405 & 345 & 190 & 340 & 385 & 315 & 445 & 82 & 142 & 116 \\
\hline Co & 41 & 44 & 30 & 43 & 49 & 36 & 48 & 29 & 43 & 36 \\
\hline $\mathrm{Cr}$ & 80 & 94 & 57 & 995 & 310 & 445 & 68 & 123 & 215 & 228 \\
\hline Sc & 7 & 20 & 12 & 31 & 10 & 19 & 10 & 43 & 30 & 37 \\
\hline S & 210 & 1020 & 340 & 410 & 255 & 240 & 400 & 350 & 415 & 370 \\
\hline As & 41 & 4 & 10.5 & 1.55 & 1.63 & 4.8 & 9.3 & 6.4 & 9.02 & 8.7 \\
\hline $\mathrm{Sb}$ & 0.92 & 0.10 & 0.03 & 0.04 & 0.04 & 0.82 & 2.40 & 3.50 & 0.76 & 1.01 \\
\hline $\mathrm{Cl}$ & 147 & 202 & 265 & 1235 & 165 & 185 & 116 & 133 & $<43$ & $<38$ \\
\hline $\mathrm{F}$ & 200 & 200 & 375 & 180 & 220 & 490 & 210 & 305 & $<14$ & $<60$ \\
\hline
\end{tabular}

Table 3 (continued).

\begin{tabular}{|c|c|c|c|c|c|c|c|}
\hline Sample no.: & 137 & 138 & 139 & 141 & 2 & 7 & GABI \\
\hline Rock name: & $\mathrm{Gb}$ & $\mathrm{Gb}$ & OIGN & GbNo & D & M-D & OlGb \\
\hline B & 5 & 5 & 5 & 4 & 3 & 5 & 4 \\
\hline $\mathrm{Li}$ & 7 & 7 & 8 & 7 & 9 & 11 & 9 \\
\hline $\mathrm{Rb}$ & $\sim 1$ & -1 & $\sim 1$ & -1 & 1 & & - \\
\hline $\mathrm{Sr}$ & 132 & 145 & 144 & 118 & 266 & 319 & 112 \\
\hline $\mathrm{Ba}$ & 11 & 5.7 & 6.8 & 12.2 & 20.4 & 19 & 19 \\
\hline $\mathrm{Ga}$ & 12 & 8 & $13^{0}$ & 14 & 12 & 10 & 11 \\
\hline $\mathrm{Pb}$ & 5 & - & $<4$ & 4 & 3 & $<2$ & 4 \\
\hline $\mathrm{Cu}$ & 69 & 67 & 63 & 41 & 21 & 8 & 93 \\
\hline $\mathrm{Zn}$ & 37 & 36 & 31 & 38 & 64 & 88 & 22 \\
\hline $\mathrm{Y}$ & 11 & 10 & $<2$ & 14 & 21 & 20 & 6 \\
\hline $\mathrm{Nb}$ & $\sim 1$ & $\leq 1$ & $<1$ & $\sim 2$ & 3 & 2 & $<1$ \\
\hline $\mathrm{Zr}$ & 19 & 15 & 11 & 32 & 69 & 66 & 14 \\
\hline $\mathrm{V}$ & 170 & 168 & 51 & 178 & 166 & 174 & 110 \\
\hline $\mathrm{Ni}$ & 103 & 124 & 220 & 101 & 230 & 285 & 268 \\
\hline Co & 38 & 26 & 42 & 30 & 46 & 43 & 36 \\
\hline $\mathrm{Cr}$ & 212 & 350 & 445 & 525 & 390 & 430 & 1790 \\
\hline $\mathrm{Sc}$ & 50 & 47 & 10 & 42 & 35 & 34 & 30 \\
\hline $\mathrm{s}$ & 415 & 560 & 390 & 325 & 72 & 56 & 370 \\
\hline As & 63 & 5.6 & 12.7 & 8.3 & 6.4 & 13.8 & 10 \\
\hline $\mathrm{Sb}$ & 0.83 & 0.04 & 0.03 & 0.02 & 0.73 & 0.03 & 0.08 \\
\hline $\mathrm{Cl}$ & $<42$ & $<23$ & $<57$ & $<38$ & 180 & $<71$ & $<39$ \\
\hline $\mathrm{F}$ & 100 & 285 & 110 & 590 & 100 & - & 420 \\
\hline
\end{tabular}

Notes: $\mathrm{GbNo}=$ gabbronorite, $\mathrm{OIGb}=$ olivine gabbro, $\mathrm{OIGN}=$ olivine gabbronorite, $\mathrm{Gb}=$ gabbro, $\mathrm{TR}=$ troctolite, $\mathrm{OlNo}=$ olivine norite, $\mathrm{D}=$ diabase, $\mathrm{M}-\mathrm{D}=$ metadiabase,$-=$ not detected. 
Table 4. CIPW norm of Leg 153 gabbros.

\begin{tabular}{|c|c|c|c|c|c|c|c|c|c|c|}
\hline Sample no.: & 100 & 103 & 104 & 142 & 108 & 109 & 113 & 115 & 118 & 119 \\
\hline Rock name: & GbNo & GbNo & $\mathrm{OlGb}$ & OlGN & OlGb & OlGb & GbNo & OIGN & $\mathrm{Gb}$ & OlGb \\
\hline Or & 0.26 & 0.27 & 0.24 & 0.28 & 0.14 & 0.19 & 0.17 & 0.17 & 0.23 & 0.20 \\
\hline $\mathrm{Ab}$ & 27.95 & 22.82 & 19.77 & 19.03 & 15.87 & 15.92 & 19.35 & 18.50 & 19.75 & 18.88 \\
\hline An & 30.24 & 33.58 & 36.79 & 43.05 & 42.15 & 39.48 & 33.46 & 44.21 & 35.36 & 40.81 \\
\hline Opx & 17.63 & 10.58 & 6.60 & 7.10 & 5.44 & 5.47 & 13.49 & 12.79 & 7.67 & 6.64 \\
\hline $\mathrm{Cpx}$ & 19.53 & 24.15 & 20.98 & 6.93 & 23.24 & 23.90 & 24.55 & 14.21 & 29.26 & 21.24 \\
\hline ol & 2.45 & 6.55 & 13.60 & 22.03 & 11.41 & 13.12 & 6.99 & 8.84 & 6.07 & 10.78 \\
\hline $\mathrm{Cm}$ & 0.02 & 0.04 & 0.21 & 0.05 & 0.43 & 0.41 & 0.08 & 0.11 & 0.08 & 0.12 \\
\hline $\mathrm{Mt}$ & 1.05 & 0.90 & 1.02 & 1.11 & 0.65 & 0.71 & 1.06 & 0.69 & 0.76 & 0.75 \\
\hline II & 0.83 & 0.91 & 0.73 & 0.37 & 0.62 & 0.73 & 0.79 & 0.43 & 0.77 & 0.54 \\
\hline Ap & 0.04 & 0.20 & 0.06 & 0.05 & 0.05 & 0.07 & 0.06 & 0.05 & 0.05 & 0.04 \\
\hline TTI & 28.2 & 23.1 & 20.0 & 19.3 & 16.0 & 16.1 & 19.5 & 18.7 & 20.0 & 19.1 \\
\hline An* & 52 & 60 & 65 & 69 & 73 & 73 & 63 & 70 & 64 & 68 \\
\hline Mg\# & 68.7 & 74.3 & 74.2 & 75.6 & 82.6 & 82.2 & 72.7 & 80.5 & 77.0 & 78.7 \\
\hline $\mathrm{Ni} / \mathrm{Co}$ & 1.94 & 2.84 & 4.88 & 5.77 & 7.43 & 8.33 & 3.97 & 5.97 & 4.46 & 5.38 \\
\hline $\mathrm{Cr} / \mathrm{Ni}$ & 1.06 & 1.67 & 4.95 & 0.75 & 7.56 & 6.75 & 2.85 & 2.42 & 2.84 & 2.55 \\
\hline $\mathrm{Cr} / \mathrm{Y}$ & 9.2 & 13.8 & 120 & 56 & 220 & 155 & 38 & 105 & 44 & 89 \\
\hline $\mathrm{Ti} / \mathrm{Cr}$ & 35 & 15.7 & 2.36 & 5.07 & 0.98 & 1.23 & 6.4 & 2.54 & 6.75 & 3.14 \\
\hline $\mathrm{V} / \mathrm{Cr}$ & 2.43 & 0.90 & 0.13 & 0.24 & 0.06 & 0.07 & 0.44 & 0.17 & 0.52 & 0.21 \\
\hline $\mathrm{Cu} / \mathrm{Zn}$ & 0.69 & 2.0 & 2.09 & 1.44 & 3.67 & 3.37 & 2.0 & 2.7 & 2.03 & 3.32 \\
\hline
\end{tabular}

Table 4 (continued).

\begin{tabular}{|c|c|c|c|c|c|c|c|c|c|c|}
\hline Sample no.: & 121 & 122 & 123 & 124 & 126 & 127 & 129 & 130 & 133 & 134 \\
\hline Rock name: & TR & TR & OlNo & OlNo & OlGN & OlNo & OIGN & OlNo & GbNo & OlGb \\
\hline Or & 0.37 & 0.34 & 0.35 & 0.37 & 0.38 & 0.30 & 0.34 & 0.49 & 0.37 & 0.28 \\
\hline $\mathrm{Ab}$ & 12.52 & 16.26 & 15.18 & 22.50 & 16.77 & 16.19 & 16.58 & 13.26 & 25.74 & 22.71 \\
\hline An & 44.69 & 52.48 & 39.61 & 48.85 & 42.19 & 48.55 & 43.92 & 43.42 & 30.16 & 39.21 \\
\hline Opx & 6.07 & 4.97 & 19.08 & 9.67 & 9.54 & 10.08 & 11.36 & 15.05 & 13.14 & 7.43 \\
\hline Cpx & 3.81 & 2.23 & 6.11 & 4.39 & 12.30 & 5.36 & 10.28 & 2.14 & 23.14 & 16.53 \\
\hline Ol & 31.07 & 22.50 & 16.49 & 10.98 & 15.85 & 17.95 & 15.72 & 22.94 & 5.52 & 12.18 \\
\hline $\mathrm{Cm}$ & 0.07 & 0.02 & 0.02 & 0.01 & 0.21 & 0.07 & 0.10 & 0.01 & 0.03 & 0.05 \\
\hline $\mathrm{Mt}$ & 0.95 & 0.78 & 1.53 & 0.99 & 1.09 & 0.86 & 0.85 & 1.19 & 1.08 & 0.98 \\
\hline II & 0.37 & 0.32 & 1.26 & 1.45 & 1.23 & 0.55 & 0.74 & 1.38 & 0.75 & 0.58 \\
\hline Ap & 0.08 & 0.10 & 0.37 & 0.79 & 0.44 & 0.09 & 0.11 & 0.12 & 0.07 & 0.05 \\
\hline TTI & 12.9 & 16.6 & 15.5 & 22.9 & 17.2 & 16.5 & 16.9 & 13.8 & 26.1 & 23.0 \\
\hline An* & 78 & 76 & 72 & 68 & 72 & 75 & 73 & 77 & 54 & 63 \\
\hline $\mathrm{Mg} \#$ & 83.4 & 81.5 & 69.4 & 67.7 & 74.5 & 79.8 & 80.1 & 78.3 & 69.3 & 72.5 \\
\hline $\mathrm{Ni} / \mathrm{Co}$ & 12 & 9.9 & 7.8 & 6.33 & 7.9 & 7.86 & 8.75 & 9.3 & 2.83 & 3.30 \\
\hline $\mathrm{Cr} / \mathrm{Ni}$ & 0.60 & 0.20 & 0.27 & 0.46 & 2.93 & 0.80 & 1.41 & 0.15 & 1.50 & 1.51 \\
\hline $\mathrm{Cr} / \mathrm{Y}$ & 38 & 8.0 & 3.62 & 1.90 & 36 & 19 & 25 & 5.67 & 7.7 & 27 \\
\hline $\mathrm{Ti} / \mathrm{Cr}$ & 3.35 & 12.8 & 42 & 79 & 3.80 & 5.42 & 5.12 & 62 & 19 & 8.4 \\
\hline $\mathrm{V} / \mathrm{Cr}$ & 0.12 & 0.34 & 0.81 & 1.84 & 0.12 & 0.17 & 0.20 & 1.18 & 1.33 & 0.51 \\
\hline $\mathrm{Cu} / \mathrm{Zn}$ & 1.23 & 0.88 & 0.92 & 0.64 & 1.03 & 0.54 & 0.45 & 0.56 & 1.58 & 1.46 \\
\hline
\end{tabular}

Table 4 (continued).

\begin{tabular}{|c|c|c|c|c|c|c|c|c|}
\hline Sample no.: & 136 & 137 & 138 & 139 & 141 & 2 & 7 & GABI \\
\hline Rock name: & GbNo & Gb & $\mathrm{Gb}$ & OIGN & GbNo & D & M-D & OIGb \\
\hline Or & 0.21 & 0.22 & 0.21 & 0.20 & 0.26 & 0.26 & 0.34 & 0.17 \\
\hline $\mathrm{Ab}$ & 22.35 & 23.15 & 21.12 & 20.29 & 19.85 & 20.34 & 25.80 & 15.10 \\
\hline An & 36.26 & 32.11 & 30.94 & 50.12 & 33.02 & 35.56 & 31.18 & 41.40 \\
\hline Opx & 9.98 & 4.54 & 6.84 & 5.04 & 12.34 & 8.40 & 5.85 & 2.55 \\
\hline $\mathrm{Cpx}$ & 21.91 & 27.53 & 29.54 & 8.49 & 28.57 & 12.34 & 5.66 & 24.13 \\
\hline Ol & 7.59 & 10.53 & 9.48 & 14.65 & 3.93 & 19.63 & 27.70 & 15.05 \\
\hline $\mathrm{Cm}$ & 0.05 & 0.05 & 0.08 & 0.10 & 0.11 & 0.08 & 0.09 & 0.40 \\
\hline $\mathrm{Mt}$ & 0.97 & 1.01 & 0.99 & 0.73 & 0.89 & 1.29 & 1.34 & 0.66 \\
\hline II & 0.64 & 0.81 & 0.75 & 0.33 & 0.93 & 1.90 & 1.84 & 0.50 \\
\hline Ap & 0.04 & 0.05 & 0.05 & 0.05 & 0.10 & 0.20 & 0.20 & 0.04 \\
\hline TTI & 22.6 & 23.4 & 21.3 & 20.5 & 20.1 & 20.6 & 26.1 & 15.3 \\
\hline $\mathrm{An*}$ & 62 & 58 & 59 & 71 & 62 & 64 & 55 & 73 \\
\hline $\mathrm{Mg} \#$ & 72.0 & 71.9 & 73.8 & 77.2 & 74.8 & 72.8 & 74.4 & 83.0 \\
\hline $\mathrm{Ni} / \mathrm{Co}$ & 3.22 & 2.71 & 4.77 & 5.24 & 3.57 & 5.0 & 6.63 & 7.44 \\
\hline $\mathrm{Cr} / \mathrm{Ni}$ & 1.97 & 2.06 & 2.82 & 2.02 & 5.20 & 1.70 & 1.51 & 6.68 \\
\hline $\mathrm{Cr} / \mathrm{Y}$ & 25 & 19 & 35 & $>220$ & 37 & 18.6 & 21.5 & 298 \\
\hline $\mathrm{Ti} / \mathrm{Cr}$ & 8.68 & 11.9 & 6.69 & 2.29 & 5.49 & 14.8 & 12.8 & 0.87 \\
\hline $\mathrm{V} / \mathrm{Cr}$ & 0.57 & 0.80 & 0.48 & 0.11 & 0.34 & 0.43 & 0.40 & 0.061 \\
\hline $\mathrm{Cu} / \mathrm{Zn}$ & 1.65 & 1.86 & 1.86 & 2.03 & 1.08 & 0.33 & 0.091 & 4.23 \\
\hline
\end{tabular}

Notes: $\mathrm{GbNo}=$ gabbronorite, $\mathrm{OIGb}=$ olivine gabbro, $\mathrm{OIGN}=$ olivine gabbronorite, $\mathrm{Gb}=$ gabbro, $\mathrm{TR}=$ troctolite, OINo $=$ olivine norite, $\mathrm{D}=$ diabase, $\mathrm{M}-\mathrm{D}=$ metadiabase. Or $=$ orthoclase, $\mathrm{Ab}=$ albite, $\mathrm{An}=$ anorthite, $\mathrm{Opx}=$ orthopyroxene, $\mathrm{Cpx}=$ clinopyroxene, $\mathrm{Ol}=$ olivine $\mathrm{Cm}=$ chromite, $\mathrm{Mt}=$ magnetite, $\mathrm{Il}=\mathrm{ilmenite}, \mathrm{Ap}=$ apatite; $\mathrm{TTI}, \mathrm{An} *$, and $\mathrm{Mg} \#$ are defined in the text. 
Table 5. Mean values of Leg 153 gabbro types.

\begin{tabular}{|c|c|c|c|c|c|c|}
\hline Type: & $\mathrm{Gb}$ & OlGb & GbNo & OlGN & OlNo & TR \\
\hline$n:$ & 3 & 6 & 6 & 5 & 4 & 2 \\
\hline \multicolumn{7}{|c|}{ Major-element composition (wt\%): } \\
\hline $\mathrm{SiO}_{2}$ & 50.55 & 48.95 & 51.2 & 47.75 & 46.5 & 45.85 \\
\hline $\mathrm{TiO}_{2}$ & 0.40 & 0.32 & 0.42 & 0.32 & 0.60 & 0.18 \\
\hline $\mathrm{Al}_{2} \mathrm{O}_{3}$ & 15.95 & 17.9 & 16.25 & 19.5 & 19.3 & 20.35 \\
\hline $\mathrm{Cr}_{2} \mathrm{O}_{3}$ & 0.122 & 0.178 & 0.037 & 0.077 & 0.019 & 0.031 \\
\hline $\mathrm{Fe}_{2} \mathrm{O}_{3}$ & 6.31 & 5.42 & 6.74 & 6.04 & 7.64 & 5.85 \\
\hline $\mathrm{MnO}$ & 0.122 & 0.097 & 0.132 & 0.096 & 0.120 & 0.096 \\
\hline $\mathrm{MgO}$ & 9.35 & 10.45 & 8.95 & 10.7 & 11.3 & 14.25 \\
\hline $\mathrm{NiO}$ & 0.015 & 0.029 & 0.013 & 0.035 & 0.043 & 0.062 \\
\hline $\mathrm{CaO}$ & 13.65 & 13.35 & 12.35 & 11.45 & 10.1 & 10.4 \\
\hline $\mathrm{Na}_{2} \mathrm{O}$ & 2.48 & 2.10 & 2.68 & 2.11 & 1.93 & 1.68 \\
\hline $\mathrm{K}_{2} \mathrm{O}$ & 0.038 & 0.034 & 0.033 & 0.045 & 0.062 & 0.059 \\
\hline $\mathrm{P}_{2} \mathrm{O}$ & 0.020 & 0.022 & 0.034 & 0.057 & 0.143 & 0.040 \\
\hline LOI & 0.87 & 0.98 & 0.88 & 1.86 & 2.2 & 1.35 \\
\hline sum & 99.88 & 99.83 & 99.72 & 100.04 & 99.96 & 100.20 \\
\hline \multicolumn{7}{|c|}{ Trace-element composition (ppm): } \\
\hline $\mathrm{Sr}$ & 134 & 124 & 133 & 133 & 134 & 137 \\
\hline $\mathrm{Ba}$ & 8.6 & 11.5 & 10.4 & 10.9 & 11.3 & 11.2 \\
\hline $\mathrm{Cu}$ & 67 & 88 & 64 & 61 & 46 & 51 \\
\hline $\mathrm{Zn}$ & 35 & 32 & 43 & 45 & 64 & 48 \\
\hline $\mathrm{Y}$ & 9.7 & 8.2 & 11.7 & 11.4 & 21 & 9.5 \\
\hline $\mathrm{Zr}$ & 17 & 17 & 32 & 39 & 67 & 26 \\
\hline $\mathrm{V}$ & 175 & 120 & 164 & 80 & 78 & 34 \\
\hline $\mathrm{Ni}$ & 117 & 225 & 102 & 278 & 341 & 485 \\
\hline Co & 24 & 38 & 29 & 42 & 43 & 44 \\
\hline $\mathrm{Cr}$ & 832 & 1220 & 252 & 526 & 132 & 210 \\
\hline Sc & 42 & 32 & 42 & 19 & 13 & 8 \\
\hline S & 415 & 385 & 355 & 355 & 505 & 235 \\
\hline $\mathrm{Ni} / \mathrm{Co}$ & 4.88 & 5.92 & 3.52 & 6.62 & 7.93 & 11 \\
\hline $\mathrm{Cr} / \mathrm{Ni}$ & 7.11 & 5.42 & 2.47 & 1.89 & 0.39 & 0.43 \\
\hline $\mathrm{Cr} / \mathrm{Zr}$ & 49 & 72 & 7.9 & 13.5 & 1.97 & 8.1 \\
\hline $\mathrm{Ti} / \mathrm{Cr}$ & 2.88 & 1.57 & 10 & 3.65 & 27 & 5.14 \\
\hline $\mathrm{V} / \mathrm{Cr}$ & 0.21 & 0.10 & 0.65 & 0.15 & 0.59 & 0.16 \\
\hline $\mathrm{Ti} / \mathrm{P}$ & 28 & 20 & 17 & 7.68 & 5.76 & 6.17 \\
\hline $\mathrm{Cu} / \mathrm{Zn}$ & 1.91 & 2.75 & 1.49 & 1.36 & 0.72 & 1.06 \\
\hline
\end{tabular}

Notes: $\mathrm{GbNo}=$ gabbronorite, $\mathrm{OIGb}=$ olivine gabbro, $\mathrm{OlGN}=$ olivine gabbronorite, $\mathrm{Gb}=$ gabbro, $\mathrm{TR}=$ troctolite, $\mathrm{OINo}=$ olivine norite $n=$ number of analyses.

Table 6. CIPW norms, Leg 153 gabbro types.

\begin{tabular}{ccccccc}
\hline Type: & Gb & OlGb & GbNo & OlGN & OlNo & TR \\
$n:$ & \multicolumn{1}{c}{3} & 6 & 6 & 5 & 4 & \multicolumn{1}{c}{2} \\
\hline Or & 0.22 & 0.20 & 0.20 & 0.28 & 0.39 & 0.36 \\
$\mathrm{Ab}$ & 21.31 & 18.06 & 23.09 & 18.28 & 16.82 & 14.45 \\
$\mathrm{An}$ & 32.80 & 39.97 & 32.80 & 44.66 & 45.12 & 48.64 \\
$\mathrm{Opx}$ & 6.20 & 5.42 & 12.74 & 8.96 & 13.39 & 5.53 \\
$\mathrm{Cpx}$ & 28.83 & 21.76 & 23.66 & 10.54 & 4.47 & 2.88 \\
$\mathrm{Ol}$ & 8.72 & 12.86 & 5.58 & 15.52 & 17.13 & 26.80 \\
$\mathrm{Cm}$ & 0.18 & 0.27 & 0.05 & 0.11 & 0.03 & 0.05 \\
$\mathrm{Mt}$ & 0.92 & 0.79 & 0.99 & 0.89 & 1.13 & 0.85 \\
$\mathrm{Il}$ & 0.77 & 0.62 & 0.81 & 0.62 & 1.17 & 0.35 \\
$\mathrm{Ap}$ & 0.05 & 0.05 & 0.08 & 0.14 & 0.35 & 0.09 \\
TTI & 21.5 & 18.3 & 23.3 & 18.6 & 17.2 & 14.8 \\
$\mathrm{An} *$ & 61 & 69 & 59 & 71 & 73 & 77 \\
$\mathrm{Mg} \#$ & 74.2 & 78.9 & 72.0 & 77.5 & 74.2 & 82.6 \\
\hline
\end{tabular}

Note: Abbreviations as in Table 3.

mean values from several analyses with nearly equal results of an individual grain are listed. In some cases, the analytical data of profiles through one grain are provided.

The $\mathrm{Mg}$ value $(\mathrm{Mg} \#)$ is given for the minerals olivine, orthopyroxene, clinopyroxene, and amphibole; $\mathrm{Ca} \#(=\mathrm{Ca} /[\mathrm{Ca}+\mathrm{Mg}])$ is also provided for clinopyroxenes; and the calculated anorthite content $(\mathrm{An \#})$ is presented for plagioclases.

\section{SUMMARY}

Twenty-six samples of gabbroic rocks from Leg 153 were analyzed for major-element oxides and 21 trace elements. In a limited number of thin sections, the mineral chemistries of olivine, orthopyroxene, clinopyroxene, amphibole, and plagioclase were investigated using an electron microprobe. This data report will form the basis for further work.

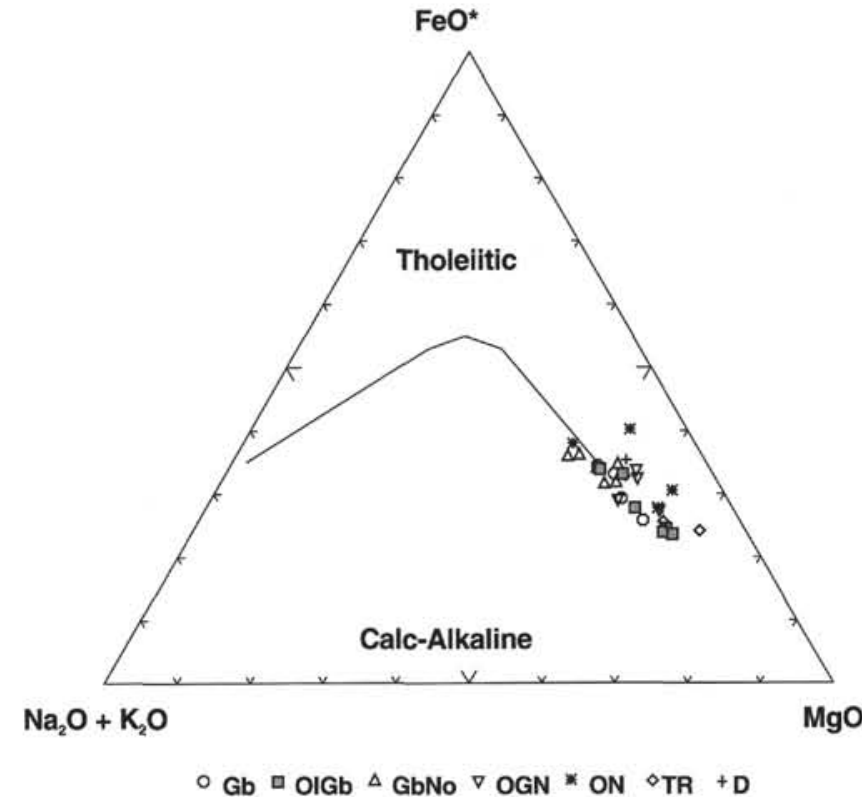

Figure 1. Projection of compositions of Leg 153 gabbros on a $\left(\mathrm{Na}_{2} \mathrm{O}+\mathrm{K}_{2} \mathrm{O}\right)$ - $\mathrm{FeO}^{*}-\mathrm{MgO}$ (= AFM) triangle, after Irvine and Baragar (1971).

\section{ACKNOWLEDGMENTS}

This work was supported by a grant from the Deutsche Forschungsgemeinschaft, Bonn. H.-J. Bernhard (Bochum), B. Knipping (Clausthal), and G. Bombach and E. Rüdiger (Freiberg) assisted with the analytical procedures. I wish to thank all these colleagues and their institutions. 


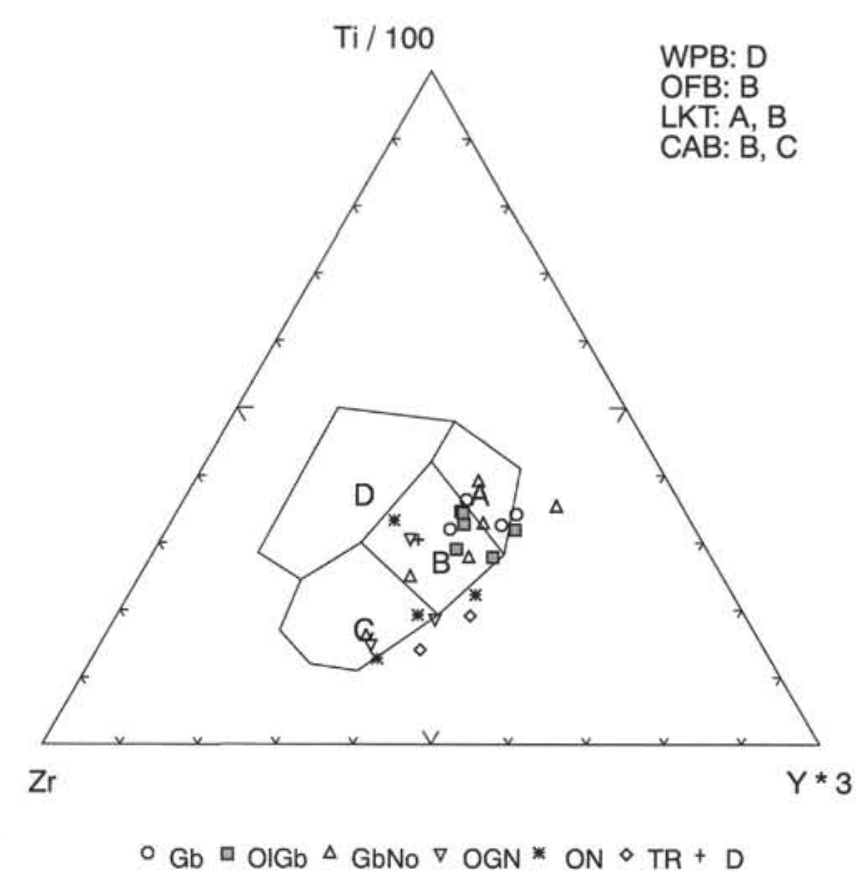

Figure 2. Compositions of Leg 153 gabbros plotted on a $\mathrm{Zr}$-Ti-Y triangle, after Pearce and Cann (1973).

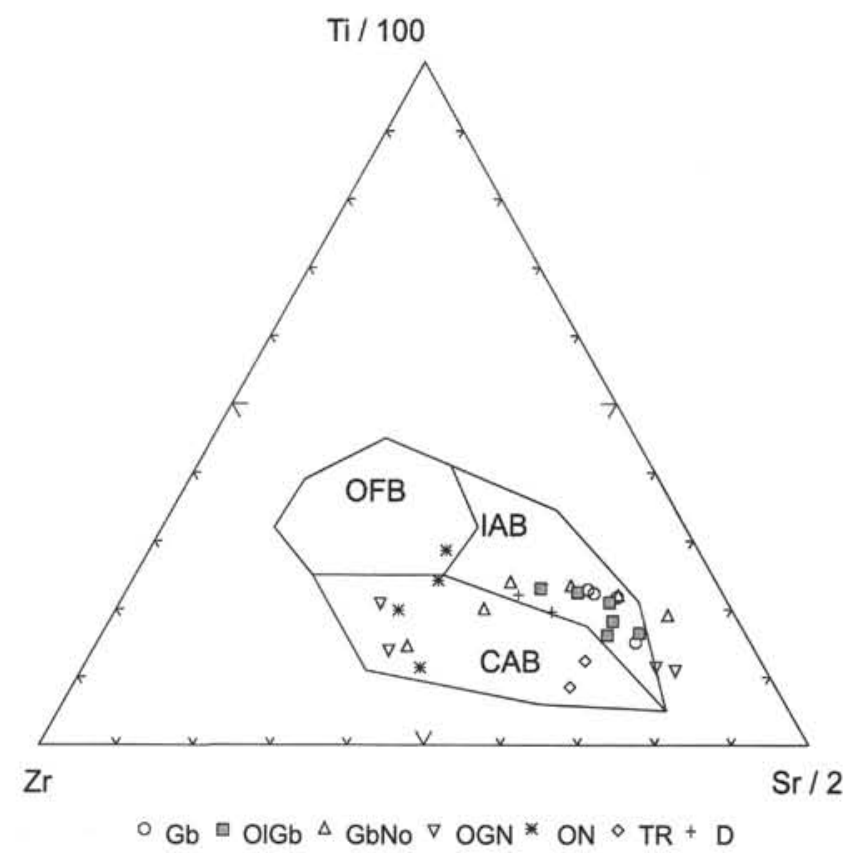

Figure 3. Compositions of Leg 153 gabbros plotted on a $\mathrm{Zr}$-Ti-Sr triangle, after Pearce and Cann (1973).

\section{REFERENCES}

Irvine, T.N., and Baragar, W.R.A., 1971. A guide to the chemical classification of the common volcanic rocks. Can. J. Earth Sci., 8:523-548.

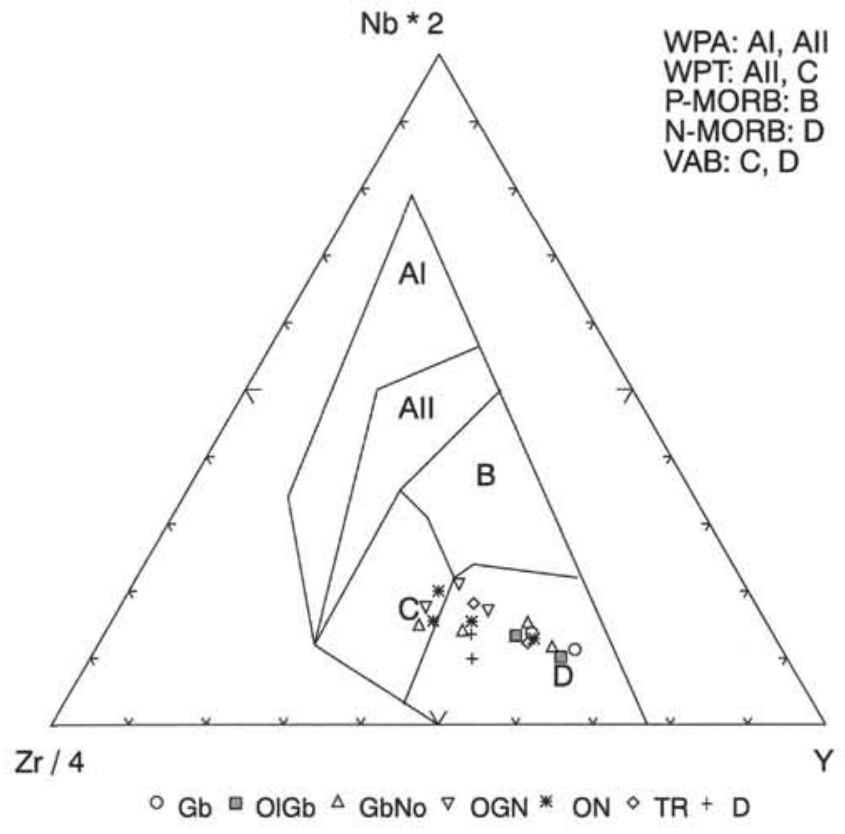

Figure 4. Compositions of Leg 153 gabbros plotted on a $\mathrm{Zr}-\mathrm{Nb}-\mathrm{Y}$ triangle, after Meschede (1986).

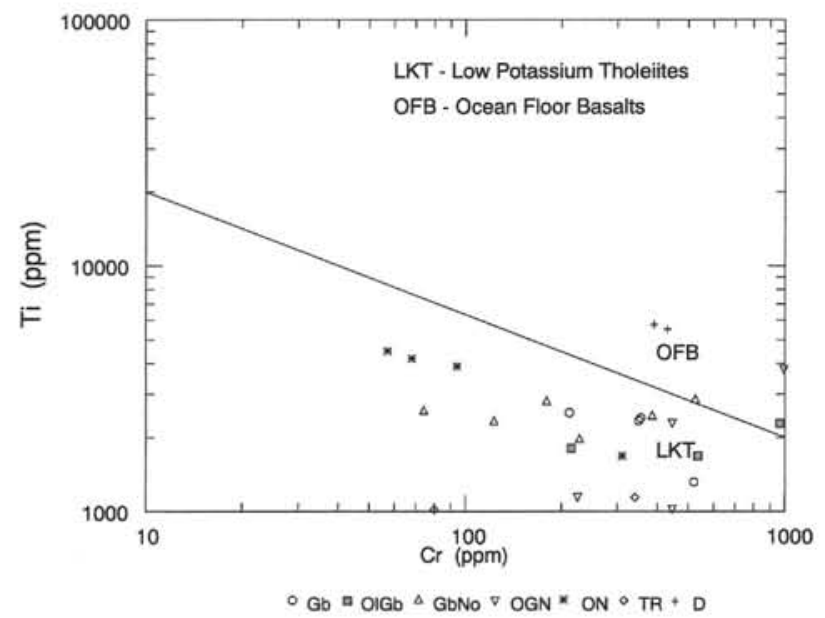

Figure 5. Compositions of Leg 153 gabbros plotted on a $\mathrm{Cr} / \mathrm{Ti}$ correlation diagram, after Pearce (1975).

Meschede, M., 1986. A method of discriminating between different types of mid-oceanic ridge basalts and continental tholeites with the $\mathrm{Nb}-\mathrm{Zr}-\mathrm{Y}$ diagram. Chem. Geol., 56:207-218.

Pearce, J.A., 1975. Basalt geochemistry used to investigate past tectonic environments on Cyprus. Tectonophysics, 25:41-67.

Pearce, J.A., and Cann, J.R., 1973. Tectonic setting of basic volcanic rocks determined using trace element analyses. Earth Planet. Sci. Lett., 19:290-300.

Date of initial receipt: 8 August 1995

Date of acceptance: 2 January 1996

Ms 153SR-031 
Table 7. Chemical composition of selected olivines.

\begin{tabular}{|c|c|c|c|c|c|c|c|c|c|c|}
\hline \multirow{3}{*}{$\begin{array}{l}\text { Sample no: } \\
\text { Rock name: } \\
n:\end{array}$} & \multicolumn{4}{|c|}{102} & \multicolumn{3}{|c|}{104} & \multicolumn{3}{|c|}{114} \\
\hline & \multicolumn{4}{|c|}{$\mathrm{Gb}$} & \multicolumn{3}{|c|}{$\mathrm{Gb}$} & \multicolumn{3}{|c|}{ OlGb } \\
\hline & 2 & 7 & 2 & 8 & 15 & 5 & 10 & 1 & 10 & 1 \\
\hline $\mathrm{SiO}_{2}$ & 35.72 & 35.93 & 36.14 & 36.16 & 36.60 & 36.81 & 37.58 & 38.48 & 38.68 & 38.7 \\
\hline $\mathrm{TiO}_{2}$ & 0.02 & 0.03 & 0.02 & 0.04 & 0.02 & 0.02 & 0.03 & - & 0.02 & 0.03 \\
\hline $\mathrm{Al}_{2} \mathrm{O}_{3}$ & 0.01 & 0.02 & 0.01 & 0.03 & 0.02 & 0.02 & 0.02 & 0.02 & 0.02 & 0.04 \\
\hline $\mathrm{Cr}_{2} \mathrm{O}_{3}$ & 0.02 & 0.01 & 0.01 & 0.02 & 0.01 & 0.02 & 0.02 & 0.01 & 0.03 & 0.04 \\
\hline $\mathrm{FeO}$ & 33.56 & 32.85 & 32.51 & 32.08 & 28.05 & 27.60 & 22.35 & 20.03 & 19.40 & 18.82 \\
\hline $\mathrm{MnO}$ & 0.52 & 0.52 & 0.51 & 0.47 & 0.41 & 0.40 & 0.33 & 0.32 & 0.30 & 0.29 \\
\hline $\mathrm{MgO}$ & 29.99 & 30.17 & 30.72 & 30.97 & 34.40 & 34.68 & 39.23 & 41.06 & 41.42 & 41.33 \\
\hline $\mathrm{NiO}$ & 0.06 & 0.09 & 0.12 & 0.08 & 0.12 & 0.11 & 0.14 & 0.13 & 0.12 & 0.22 \\
\hline $\mathrm{CaO}$ & 0.07 & 0.06 & 0.03 & 0.06 & 0.05 & 0.05 & 0.05 & 0.03 & 0.05 & 0.05 \\
\hline Total & 99.97 & 99.68 & 100.07 & 99.91 & 99.68 & 99.71 & 99.75 & 100.08 & 100.04 & 99.53 \\
\hline Mg\# & 61.4 & 62.0 & 62.7 & 63.2 & 68.6 & 69.1 & 75.8 & 78.5 & 79.2 & 79.6 \\
\hline
\end{tabular}

Table 7 (continued).

\begin{tabular}{|c|c|c|c|c|c|c|c|c|c|c|}
\hline \multirow{3}{*}{$\begin{array}{l}\text { Sample no.: } \\
\text { Rock name: } \\
n:\end{array}$} & \multicolumn{3}{|c|}{119} & \multicolumn{3}{|c|}{113} & \multicolumn{4}{|c|}{124} \\
\hline & \multicolumn{3}{|c|}{$\mathrm{OlGb}$} & \multicolumn{3}{|c|}{ GbNo } & \multicolumn{4}{|c|}{ OlNo } \\
\hline & 3 & 4 & 5 & 6 & 1 & 4 & 7 & 2 & 3 & 3 \\
\hline $\mathrm{SiO}_{2}$ & 38.52 & 38.58 & 38.60 & 38.44 & 37.23 & 37.21 & 37.19 & 38.91 & 39.00 & 38.84 \\
\hline $\mathrm{TiO}_{2}$ & 0.02 & 0.01 & 0.02 & 0.02 & 0.03 & 0.01 & 0.03 & 0.02 & 0.02 & 0.02 \\
\hline $\mathrm{Al}_{2} \mathrm{O}_{3}$ & 0.01 & 0.02 & 0.02 & 0.02 & 0.03 & 0.02 & 0.02 & 0.01 & 0.03 & 0.03 \\
\hline $\mathrm{Cr}_{2} \mathrm{O}_{3}$ & 0.01 & 0.04 & 0.01 & 0.01 & - & 0.04 & 0.02 & 0.02 & - & 0.02 \\
\hline $\mathrm{FeO}$ & 20.77 & 20.64 & 20.62 & 20.27 & 28.58 & 28.16 & 28.45 & 21.07 & 21.04 & 20.72 \\
\hline $\mathrm{MnO}$ & 0.28 & 0.32 & 0.27 & 0.32 & 0.43 & 0.44 & 0.43 & 0.34 & 0.31 & 0.33 \\
\hline $\mathrm{MgO}$ & 40.34 & 40.44 & 40.68 & 40.71 & 34.14 & 34.52 & 34.30 & 40.19 & 40.49 & 40.56 \\
\hline $\mathrm{NiO}$ & 0.15 & 0.14 & 0.14 & 0.15 & 0.02 & 0.09 & 0.05 & 0.17 & 0.17 & 0.20 \\
\hline $\mathrm{CaO}$ & 0.03 & 0.03 & 0.03 & 0.04 & 0.07 & 0.05 & 0.07 & 0.06 & 0.04 & 0.04 \\
\hline Total & 100.13 & 100.22 & 100.39 & 99.98 & 100.53 & 100.54 & 100.55 & 100.79 & 101.10 & 100.76 \\
\hline Mg\# & 77.6 & 77.7 & 77.9 & 78.2 & 68.0 & 68.6 & 68.2 & 77.3 & 77.5 & 77.7 \\
\hline
\end{tabular}

Table 7 (continued).

\begin{tabular}{|c|c|c|c|c|c|c|c|c|c|c|}
\hline \multirow{3}{*}{$\begin{array}{l}\text { Sample no.: } \\
\text { Rock name: } \\
n:\end{array}$} & \multicolumn{6}{|c|}{122} & \multicolumn{4}{|c|}{121} \\
\hline & \multicolumn{6}{|c|}{ TR } & \multicolumn{4}{|c|}{ TR } \\
\hline & 3 & 6 & 5 & 5 & 10 & 5 & 10 & 10 & 10 & 10 \\
\hline $\mathrm{SiO}_{2}$ & 40.07 & 39.77 & 39.54 & 39.81 & 39.78 & 39.83 & 39.84 & 39.75 & 39.93 & 39.93 \\
\hline $\mathrm{TiO}_{2}$ & 0.03 & 0.02 & 0.02 & 0.01 & 0.02 & 0.01 & 0.02 & 0.03 & 0.03 & 0.03 \\
\hline $\mathrm{Al}_{2} \mathrm{O}_{3}$ & 0.01 & 0.02 & 0.02 & 0.02 & 0.02 & 0.01 & 0.01 & 0.01 & 0.01 & 0.02 \\
\hline $\mathrm{Cr}_{2} \mathrm{O}_{3}$ & 0.04 & 0.02 & 0.01 & 0.01 & 0.01 & 0.02 & 0.02 & 0.01 & 0.02 & 0.02 \\
\hline $\mathrm{FeO}^{3}$ & 14.32 & 15.90 & 16.02 & 15.46 & 15.26 & 15.13 & 14.28 & 14.26 & 14.19 & 14.31 \\
\hline $\mathrm{MnO}$ & 0.25 & 0.27 & 0.29 & 0.25 & 0.24 & 0.26 & 0.24 & 0.24 & 0.25 & 0.24 \\
\hline $\mathrm{MgO}$ & 45.33 & 44.53 & 44.17 & 44.57 & 44.68 & 44.62 & 45.42 & 45.47 & 45.51 & 45.50 \\
\hline $\mathrm{NiO}$ & 0.17 & 0.20 & 0.21 & 0.19 & 0.21 & 0.23 & 0.21 & 0.23 & 0.18 & 0.22 \\
\hline $\mathrm{CaO}$ & 0.03 & 0.03 & 0.04 & 0.03 & 0.04 & 0.04 & 0.05 & 0.05 & 0.04 & 0.04 \\
\hline Total & 100.25 & 100.76 & 100.32 & 100.35 & 100.26 & 100.15 & 100.07 & 100.05 & 100.16 & 100.31 \\
\hline $\mathrm{Mg \#}$ & 84.9 & 83.3 & 83.1 & 83.7 & 83.9 & 84.0 & 85.0 & 85.0 & 85.1 & 85.0 \\
\hline
\end{tabular}

Notes: Abbreviations as in Table $1 ; n=$ number of analyses; $-=$ not detected. 
Table 8. Chemical composition of selected orthopyroxenes.

\begin{tabular}{|c|c|c|c|c|c|c|c|c|c|c|}
\hline \multirow{4}{*}{$\begin{array}{l}\text { Sample no.: } \\
\text { Rock name: } \\
n:\end{array}$} & \multicolumn{3}{|c|}{102} & \multicolumn{7}{|c|}{118} \\
\hline & \multirow{2}{*}{\multicolumn{3}{|c|}{$\mathrm{Gb}$}} & \multicolumn{7}{|c|}{$\mathrm{Gb}$} \\
\hline & & & & \multicolumn{7}{|c|}{ Within clinopyroxene } \\
\hline & 2 & 1 & 6 & 4 & 2 & 3 & 3 & 2 & 2 & 2 \\
\hline $\mathrm{SiO}_{2}$ & 52.28 & 52.76 & 52.83 & 53.43 & 53.73 & 53.40 & 53.58 & 53.75 & 53.57 & 53.89 \\
\hline $\mathrm{TiO}_{2}$ & 0.27 & 0.31 & $\begin{array}{r}J 2.03 \\
0.32\end{array}$ & $\begin{array}{r}53.43 \\
0.36\end{array}$ & 0.36 & 0.41 & 0.38 & 0.38 & 0.31 & 0.31 \\
\hline $\mathrm{Al}_{2} \mathrm{O}_{3}$ & 0.95 & 1.09 & 1.12 & 1.28 & 1.24 & 1.29 & 1.21 & 1.14 & 1.36 & 1.29 \\
\hline $\mathrm{Cr}_{2} \mathrm{O}_{3}$ & 0.01 & - & 0.03 & 0.05 & 0.09 & 0.04 & 0.07 & 0.08 & 0.04 & 0.03 \\
\hline $\mathrm{FeO}$ & 19.53 & 19.33 & 19.85 & 16.34 & 16.10 & 16.28 & 16.49 & 16.82 & 14.95 & 15.10 \\
\hline $\mathrm{MnO}$ & 0.50 & 0.47 & 0.53 & 0.34 & 0.37 & 0.38 & 0.37 & 0.43 & 0.38 & 0.28 \\
\hline $\mathrm{MgO}$ & 23.82 & 23.88 & 23.59 & 26.34 & 26.70 & 26.48 & 26.44 & 26.18 & 27.58 & 27.38 \\
\hline $\mathrm{NiO}$ & 0.01 & - & 0.06 & 0.04 & 0.05 & 0.02 & 0.06 & 0.02 & 0.07 & 0.04 \\
\hline $\mathrm{CaO}$ & 1.15 & 1.35 & 1.38 & 1.08 & $\begin{array}{l}0.07 \\
1.07\end{array}$ & 1.17 & 1.16 & 1.06 & 1.10 & 1.27 \\
\hline $\mathrm{Na}_{2} \mathrm{O}$ & 0.01 & 0.01 & 0.01 & 0.03 & 0.02 & 0.02 & 0.01 & 0.02 & - & 0.02 \\
\hline Total & 98.53 & 99.20 & 99.72 & 99.39 & 99.72 & 99.49 & 99.67 & 99.88 & 99.36 & 99.61 \\
\hline $\mathrm{Mg \#}$ & 68.0 & 68.8 & 67.9 & 74.2 & 74.7 & 74.4 & 74.1 & 73.5 & 76.7 & 76.4 \\
\hline
\end{tabular}

Table 8 (continued).

\begin{tabular}{|c|c|c|c|c|c|c|c|c|c|}
\hline Sample no.: & 104 & 114 & \multicolumn{2}{|c|}{116} & \multicolumn{5}{|c|}{119} \\
\hline Rock name: & $\mathrm{OlGb}$ & OlGb & \multirow{2}{*}{\multicolumn{2}{|c|}{ OlGb }} & \multirow{2}{*}{\multicolumn{5}{|c|}{ OlGb }} \\
\hline \multirow[b]{2}{*}{$n:$} & \multirow[b]{2}{*}{1} & \multirow[b]{2}{*}{2} & & & & & & oxene & \\
\hline & & & 6 & 2 & 5 & 4 & 3 & 3 & 2 \\
\hline $\mathrm{SiO}_{2}$ & 53.54 & 55.20 & 54.02 & 54.25 & 54.38 & 54.80 & 54.39 & 54.33 & 54.04 \\
\hline $\mathrm{TiO}_{2}$ & 0.23 & 0.21 & 0.21 & 0.21 & 0.24 & 0.34 & 0.30 & 0.26 & 0.31 \\
\hline $\mathrm{Al}_{2} \mathrm{O}_{3}$ & 0.95 & 1.04 & 2.50 & 2.49 & 2.36 & 1.38 & 1.21 & 1.32 & 1.52 \\
\hline $\mathrm{Cr}_{2} \mathrm{O}_{3}$ & 0.11 & 0.14 & 0.13 & 0.10 & 0.14 & 0.06 & 0.13 & 0.09 & 0.08 \\
\hline $\mathrm{FeO}$ & 16.75 & 12.13 & 11.93 & 11.98 & 12.00 & 12.92 & 14.01 & 14.53 & 14.96 \\
\hline $\mathrm{MnO}$ & 0.49 & 0.24 & 0.28 & 0.24 & 0.27 & 0.30 & 0.33 & 0.34 & 0.34 \\
\hline $\mathrm{MgO}$ & 26.54 & 30.05 & 29.24 & 29.20 & 29.34 & 29.12 & 28.02 & 27.93 & 27.16 \\
\hline $\mathrm{NiO}$ & 0.03 & 0.03 & 0.05 & 0.04 & 0.04 & 0.05 & 0.06 & 0.06 & 0.05 \\
\hline $\mathrm{CaO}$ & 0.81 & 0.88 & 0.87 & 0.98 & 0.96 & 0.92 & 1.08 & 0.98 & 1.27 \\
\hline $\mathrm{Na}_{2} \mathrm{O}$ & - & 0.01 & 0.01 & 0.03 & 0.01 & 0.01 & 0.01 & 0.02 & 0.02 \\
\hline Total & 99.45 & 99.93 & 99.24 & 99.52 & 99.74 & 99.90 & 99.54 & 99,86 & 99.75 \\
\hline Mg\# & 73.8 & 81.5 & 81.4 & 81.3 & 81.4 & 80.1 & 78.1 & 77.4 & 76.4 \\
\hline
\end{tabular}

Table 8 (continued).

\begin{tabular}{|c|c|c|c|c|c|c|c|c|c|c|}
\hline \multirow{3}{*}{$\begin{array}{l}\text { Sample no.: } \\
\text { Rock name: } \\
n:\end{array}$} & \multicolumn{5}{|c|}{123} & \multirow{2}{*}{$\begin{array}{c}124 \\
\text { OINo }\end{array}$} & \multirow{2}{*}{$\begin{array}{l}121 \\
\text { TR } \\
\end{array}$} & \multicolumn{3}{|c|}{122} \\
\hline & \multicolumn{5}{|c|}{ OINo } & & & \multicolumn{3}{|c|}{ TR } \\
\hline & 2 & 5 & 3 & 5 & 3 & 6 & 3 & 2 & 3 & 3 \\
\hline $\mathrm{SiO}_{2}$ & 51.44 & 52.25 & 52.04 & 52.45 & 52.22 & 55.43 & 56.01 & 55.91 & 55.42 & 55.85 \\
\hline $\mathrm{TiO}_{2}$ & 0.23 & 0.20 & 0.30 & 0.43 & 0.41 & 0.23 & 0.33 & 0.42 & 0.50 & 0.34 \\
\hline $\mathrm{Al}_{2} \mathrm{O}_{3}$ & 0.65 & 0.44 & 0.95 & 1.32 & 1.47 & 1.07 & 1.25 & 1.46 & 1.53 & 1.44 \\
\hline $\mathrm{Cr}_{2} \mathrm{O}_{3}$ & 0.02 & 0.03 & 0.01 & 0.04 & 0.02 & 0.02 & 0.16 & 0.18 & 0.20 & 0.21 \\
\hline $\mathrm{FeO}$ & 27.81 & 26.76 & 24.70 & 22.78 & 21.99 & 12.77 & 9.06 & 8.97 & 10.20 & 9.27 \\
\hline $\mathrm{MnO}$ & 0.89 & 0.83 & 0.62 & 0.59 & 0.53 & 0.36 & 0.24 & 0.25 & 0.29 & 0.21 \\
\hline $\mathrm{MgO}$ & 18.03 & 19.30 & 20.29 & 21.70 & 21.45 & 29.46 & 31.98 & 31.94 & 30.92 & 31.74 \\
\hline $\mathrm{NiO}$ & 0.03 & 0.05 & 0.05 & 0.02 & 0.05 & 0.05 & 0.07 & 0.05 & 0.05 & 0.03 \\
\hline $\mathrm{CaO}$ & 1.14 & 0.99 & 1.50 & 1.32 & 2.20 & 0.85 & 0.72 & 0.81 & 1.19 & 0.82 \\
\hline \multirow{2}{*}{$\mathrm{Na}_{2} \mathrm{O}$} & - & 0.02 & 0.04 & 0.03 & 0.04 & 0.02 & 0.01 & 0.03 & 0.01 & 0.01 \\
\hline & 100.24 & 100.87 & 100.5 & 100.68 & 100.38 & 100.26 & 99.83 & 100.02 & 100.31 & 99.92 \\
\hline Mg\# & 53.6 & 56.2 & 59.4 & 62.9 & 63.5 & 80.4 & 86.3 & 86.4 & 84.4 & 85.9 \\
\hline
\end{tabular}

Note: Abbreviations as in Table $4 ;-=$ not detected. 
Table 9. Chemical composition of selected clinopyroxenes.

\begin{tabular}{|c|c|c|c|c|c|c|c|c|c|}
\hline \multirow{3}{*}{$\begin{array}{l}\text { Sample no.: } \\
\text { Rock name: } \\
n:\end{array}$} & \multicolumn{3}{|c|}{102} & \multicolumn{6}{|c|}{110} \\
\hline & \multicolumn{3}{|c|}{$\mathrm{Gb}$} & \multicolumn{6}{|c|}{$\mathrm{Gb}$} \\
\hline & 4 & 2 & 3 & 3 & 2 & 5 & 4 & 10 & 7 \\
\hline $\mathrm{SiO}_{2}$ & 50.83 & 50.90 & 50.65 & 52.94 & 53.57 & 51.59 & 52.10 & 52.35 & 52.46 \\
\hline $\mathrm{TiO}_{2}$ & 0.82 & 0.75 & 0.79 & 0.24 & 0.08 & 0.90 & 0.84 & 0.58 & 0.59 \\
\hline $\mathrm{Al}_{2} \mathrm{O}_{3}$ & 2.70 & 2.27 & 2.37 & 0.91 & 0.76 & 2.51 & 2.86 & 2.83 & 2.92 \\
\hline $\mathrm{Cr}_{2} \mathrm{O}_{3}$ & 0.02 & 0.03 & 0.05 & 0.02 & 0.03 & 0.08 & 0.11 & 0.17 & 0.15 \\
\hline $\mathrm{FeO}$ & 9.20 & 9.30 & 9.44 & 8.47 & 7.51 & 9.22 & 6.27 & 6.53 & 5.91 \\
\hline $\mathrm{MnO}$ & 0.25 & 0.25 & 0.31 & 0.30 & 0.29 & 0.23 & 0.19 & 0.19 & 0.19 \\
\hline $\mathrm{MgO}$ & 14.66 & 14.25 & 14.19 & 14.45 & 14.93 & 14.58 & 15.22 & 16.52 & 16.92 \\
\hline $\mathrm{NiO}$ & 0.02 & 0.03 & 0.01 & 0.01 & 0.03 & 0.04 & 0.02 & 0.02 & 0.02 \\
\hline $\mathrm{CaO}$ & 20.50 & 21.50 & 21.37 & 22.96 & 23.00 & 20.93 & 22.34 & 20.76 & 20.85 \\
\hline $\mathrm{Na}_{2} \mathrm{O}$ & 0.52 & 0.44 & 0.43 & 0.20 & 0.16 & 0.40 & 0.47 & 0.36 & 0.36 \\
\hline $\mathrm{K}_{2} \mathrm{O}$ & - & - & 0.01 & - & - & 0.01 & - & - & - \\
\hline Total & 99.52 & 99.72 & 99.62 & 100.49 & 100.36 & 100.49 & 100.42 & 100.31 & 100.37 \\
\hline $\mathrm{Mg \#}$ & 73.9 & 73.2 & 72.8 & 75.3 & 78.0 & 73.8 & 81.2 & 81.9 & 83.6 \\
\hline Ca\# & 50.0 & 50.7 & 52.0 & 53.3 & 53.1 & 50.6 & 51.3 & 47.5 & 47.0 \\
\hline
\end{tabular}

Table 9 (continued).

\begin{tabular}{|c|c|c|c|c|c|c|c|c|c|}
\hline \multirow{4}{*}{$\begin{array}{l}\text { Sample no.: } \\
\text { Rock name: } \\
n:\end{array}$} & \multicolumn{9}{|c|}{118} \\
\hline & \multicolumn{9}{|c|}{$\mathrm{Gb}$} \\
\hline & & \multicolumn{4}{|c|}{ Profile 1} & \multicolumn{4}{|c|}{ Profile 2} \\
\hline & 7 & 3 & 6 & 3 & 3 & 3 & 5 & 2 & 5 \\
\hline $\mathrm{SiO}_{2}$ & 50.74 & 51.19 & 51.18 & 51.19 & 50.52 & 51.31 & 50.69 & 50.91 & 50.82 \\
\hline $\mathrm{TiO}_{2}^{2}$ & 1.08 & 0.85 & 0.92 & 0.97 & 1.04 & 0.94 & 0.99 & 1.02 & 1.01 \\
\hline $\mathrm{Al}_{2} \mathrm{O}_{3}$ & 2.73 & 2.45 & 2.70 & 2.65 & 2.78 & 2.50 & 2.66 & 2.64 & 2.71 \\
\hline $\mathrm{Cr}_{2} \mathrm{O}_{3}$ & 0.12 & 0.12 & 0.17 & 0.06 & 0.12 & 0.10 & 0.13 & 0.16 & 0.14 \\
\hline $\mathrm{FeO}$ & 7.07 & 6.93 & 7.20 & 6.95 & 6.91 & 7.05 & 6.93 & 6.98 & 7.02 \\
\hline $\mathrm{MnO}$ & 0.23 & 0.25 & 0.23 & 0.27 & 0.21 & 0.21 & 0.22 & 0.17 & 0.23 \\
\hline $\mathrm{MgO}$ & 15.10 & 15.13 & 15.24 & 15.24 & 14.98 & 15.30 & 14.94 & 15.10 & 15.24 \\
\hline $\mathrm{NiO}$ & 0.01 & 0.02 & 0.04 & 0.02 & 0.08 & 0.06 & 0.02 & 0.02 & 0.07 \\
\hline $\mathrm{CaO}$ & 21.93 & 22.45 & 21.82 & 21.75 & 22.16 & 21.73 & 22.10 & 22.01 & 21.86 \\
\hline $\mathrm{Na}_{2} \mathrm{O}$ & 0.42 & 0.41 & 0.42 & 0.44 & 0.42 & 0.46 & 0.45 & 0.44 & 0.41 \\
\hline $\mathrm{K}_{2} \mathrm{O}$ & 0.01 & - & 0.01 & 0.01 & - & 0.01 & - & 0.02 & 0.01 \\
\hline Total & 99.44 & 99.80 & 99.93 & 99.55 & 99.22 & 99.67 & 99.15 & 99.47 & 99.52 \\
\hline $\mathrm{Mg \#}$ & 79.2 & 79.6 & 79.1 & 79.7 & 79.4 & 79.5 & 79.3 & 79.4 & 79.5 \\
\hline $\mathrm{Ca} \#$ & 58.4 & 51.6 & 50.7 & 50.4 & 51.5 & 50.5 & 51.5 & 51.2 & 50.8 \\
\hline
\end{tabular}

Table 9 (continued).

\begin{tabular}{|c|c|c|c|c|c|c|c|c|c|}
\hline Sample no:: & \multicolumn{4}{|c|}{104} & \multicolumn{5}{|c|}{114} \\
\hline \multirow[t]{3}{*}{ Rock name: } & \multicolumn{4}{|c|}{$\mathrm{OlGb}$} & \multicolumn{5}{|c|}{$\mathrm{OlGb}$} \\
\hline & \multicolumn{4}{|c|}{ Profile } & \multicolumn{5}{|c|}{ Profile } \\
\hline & 2 & 4 & 4 & 5 & 2 & 2 & 3 & 1 & 1 \\
\hline $\mathrm{SiO}_{2}$ & 51.16 & 51.29 & 51.02 & 51.34 & 52.11 & 52.00 & 52.56 & 52.62 & 52.57 \\
\hline $\mathrm{TiO}_{2}$ & 0.61 & 0.43 & 0.39 & 0.80 & 0.32 & 0.41 & 0.48 & 0.37 & 0.50 \\
\hline $\mathrm{Al}_{2} \mathrm{O}_{3}$ & 2.96 & 3.01 & 3.03 & 2.77 & 2.09 & 2.70 & 1.99 & 2.00 & 2.03 \\
\hline $\mathrm{Cr}_{2} \mathrm{O}_{3}$ & 0.54 & 0.54 & 0.56 & 0.51 & 0.23 & 0.28 & 0.31 & 0.25 & 0.26 \\
\hline $\mathrm{FeO}$ & 5.32 & 5.62 & 6.02 & 5.46 & 4.96 & 4.85 & 4.75 & 4.83 & 4.73 \\
\hline $\mathrm{MnO}$ & 0.16 & 0.17 & 0.20 & 0.18 & 0.17 & 0.18 & 0.13 & 0.20 & 0.14 \\
\hline $\mathrm{MgO}$ & 15.93 & 16.50 & 17.38 & 15.88 & 16.34 & 16.13 & 16.16 & 16.20 & 16.36 \\
\hline $\mathrm{NiO}$ & 0.08 & 0.08 & 0.06 & 0.02 & 0.04 & 0.04 & 0.05 & 0.08 & 0.04 \\
\hline $\mathrm{CaO}$ & 22.17 & 21.36 & 20.08 & 22.05 & 22.70 & 22.62 & 22.93 & 22.79 & 23.10 \\
\hline $\mathrm{Na}_{2} \mathrm{O}$ & 0.38 & 0.39 & 0.32 & 0.39 & 0.37 & 0.38 & 0.35 & 0.35 & 0.38 \\
\hline $\mathrm{K}_{2} \mathrm{O}$ & 0.01 & - & - & - & - & 0.01 & 0.01 & - & - \\
\hline Total & 99.32 & 99.39 & 99.06 & 99.40 & 99.33 & 99.60 & 99.72 & 99.69 & 100.11 \\
\hline $\mathrm{Mg \#}$ & 84.2 & 84.0 & 83.7 & 83.8 & 85.4 & 85.6 & 85.8 & 85.7 & 86.0 \\
\hline Ca\# & 50.0 & 48.2 & 45.4 & 50.0 & 50.0 & 50.2 & 50.5 & 50.3 & 50.4 \\
\hline
\end{tabular}


Table 9 (continued).

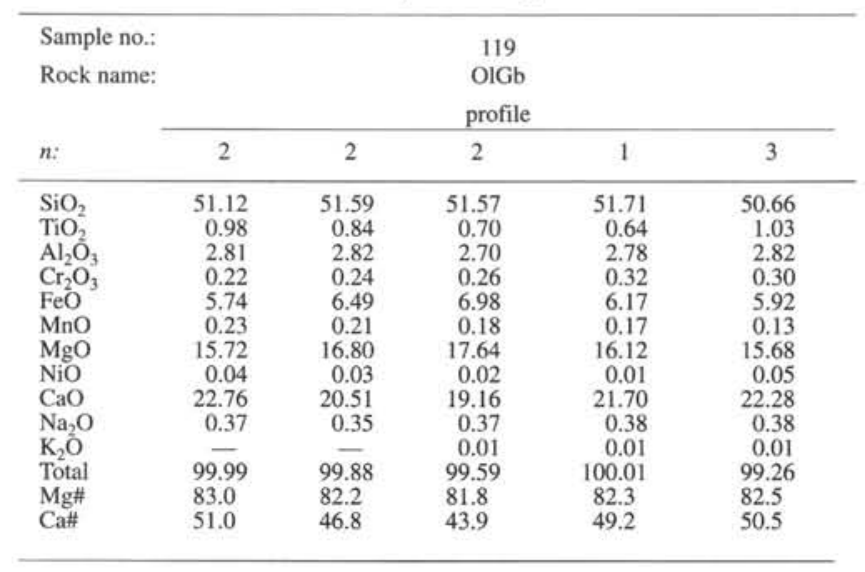

Table 9 (continued).

\begin{tabular}{|c|c|c|c|c|c|c|c|}
\hline \multirow{4}{*}{$\begin{array}{l}\text { Sample no: } \\
\text { Rock name: } \\
n:\end{array}$} & \multicolumn{7}{|c|}{113} \\
\hline & \multicolumn{7}{|c|}{ GbNo } \\
\hline & \multicolumn{5}{|c|}{ Profile } & \multicolumn{2}{|c|}{ Grains } \\
\hline & 3 & 2 & 2 & 2 & 2 & 2 & 2 \\
\hline $\mathrm{SiO}_{2}$ & 51.33 & 51.75 & 51.92 & 52.23 & 52.04 & 52.43 & 51.88 \\
\hline $\mathrm{TiO}_{2}$ & 1.03 & 0.61 & 0.54 & 0.61 & 0.57 & 0.59 & 0.64 \\
\hline $\mathrm{Al}_{2} \mathrm{O}_{3}$ & 2.78 & 2.76 & 2.82 & 2.93 & 2.88 & 2.73 & 2.79 \\
\hline $\mathrm{Cr}_{2} \mathrm{O}_{3}$ & 0.12 & 0.22 & 0.13 & 0.20 & 0.17 & 0.16 & 0.14 \\
\hline $\mathrm{FeO}$ & 7.93 & 7.77 & 6.85 & 6.42 & 5.56 & 6.51 & 6.90 \\
\hline $\mathrm{MnO}$ & 0.25 & 0.26 & 0.23 & 0.23 & 0.16 & 0.19 & 0.20 \\
\hline $\mathrm{MgO}$ & 14.78 & 16.06 & 16.04 & 16.46 & 15.86 & 17.31 & 16.75 \\
\hline $\mathrm{NiO}$ & 0.03 & 0.01 & 0.06 & 0.05 & 0.05 & 0.02 & 0.05 \\
\hline $\mathrm{CaO}$ & 21.70 & 20.61 & 21.16 & 20.97 & 22.55 & 20.09 & 20.42 \\
\hline $\mathrm{Na}_{2} \mathrm{O}$ & 0.42 & 0.44 & 0.40 & 0.41 & 0.42 & 0.36 & 0.38 \\
\hline $\mathrm{K}_{2} \mathrm{O}$ & - & 0.01 & 0.01 & - & - & - & 0.01 \\
\hline Total & 100.37 & 100.51 & 100.16 & 100.51 & 100.26 & 100.39 & 100.16 \\
\hline $\mathrm{Mg \#}$ & 76.9 & 78.6 & 80.7 & 82.1 & 83.6 & 82.6 & 81.2 \\
\hline Ca\# & 51.3 & 48.8 & 48.7 & 47.8 & 50.5 & 45.5 & 46.7 \\
\hline
\end{tabular}

Table 9 (continued).

\begin{tabular}{|c|c|c|c|c|c|c|}
\hline \multirow{3}{*}{$\begin{array}{l}\text { Sample no: } \\
\text { Rock name: } \\
n:\end{array}$} & \multicolumn{4}{|c|}{123} & \multicolumn{2}{|c|}{124} \\
\hline & \multicolumn{4}{|c|}{ OINo } & \multicolumn{2}{|c|}{ OINo } \\
\hline & 4 & 2 & 4 & 2 & 4 & 2 \\
\hline $\mathrm{SiO}_{2}$ & 51.68 & 52.00 & 52.54 & 52.36 & 52.59 & 53.38 \\
\hline $\mathrm{TiO}_{2}$ & 0.48 & 0.35 & 0.17 & 0.47 & 0.62 & 0.73 \\
\hline $\mathrm{Al}_{2} \mathrm{O}_{3}$ & 1.46 & 1.20 & 0.88 & 1.75 & 1.96 & 2.22 \\
\hline $\mathrm{Cr}_{2} \mathrm{O}_{3}$ & 0.02 & 0.01 & 0.03 & 0.03 & 0.04 & 0.08 \\
\hline $\mathrm{FeO}$ & 13.40 & 12.33 & 11.57 & 8.26 & 5.03 & 4.91 \\
\hline $\mathrm{MnO}$ & 0.46 & 0.41 & 0.42 & 0.31 & 0.18 & 0.18 \\
\hline $\mathrm{MgO}$ & 12.91 & 12.82 & 13.02 & 14.88 & 16.03 & 18.12 \\
\hline $\mathrm{NiO}$ & 0.07 & 0.02 & 0.04 & 0.01 & 0.03 & 0.05 \\
\hline $\mathrm{CaO}$ & 19.67 & 20.73 & 21.48 & 21.90 & 22.90 & 19.20 \\
\hline $\mathrm{Na}_{2} \mathrm{O}$ & 0.42 & 0.42 & 0.41 & 0.34 & 0.29 & 0.46 \\
\hline $\mathrm{K}_{2} \mathrm{O}$ & - & 0.01 & - & 0.01 & 0.01 & 0.02 \\
\hline Total & 100.57 & 100.30 & 100.56 & 100.32 & 99.68 & 99.35 \\
\hline $\mathrm{Mg \#}$ & 63.2 & 65.0 & 66.7 & 76.3 & 85.0 & 86.7 \\
\hline Ca\# & 52.3 & 53.8 & 54.3 & 51.4 & 50.7 & 43.3 \\
\hline
\end{tabular}

Table 9 (continued).

\begin{tabular}{|c|c|c|c|c|c|c|c|c|c|c|}
\hline Sample no.: & \multicolumn{3}{|c|}{121} & \multicolumn{4}{|c|}{122} & & & \\
\hline \multirow[t]{2}{*}{ Rock name: } & \multicolumn{3}{|c|}{ TR } & \multicolumn{4}{|c|}{ TR } & & & \\
\hline & \multicolumn{3}{|c|}{ Profile } & \multicolumn{4}{|c|}{ Grain, profile } & \multicolumn{3}{|c|}{ Grains } \\
\hline$n:$ & 2 & 2 & 1 & 2 & 4 & 2 & 2 & 3 & 6 & 8 \\
\hline $\mathrm{SiO}_{2}$ & 52.34 & 52.44 & 53.08 & 52.10 & 51.58 & 51.48 & 52.28 & 52.12 & 51.93 & 52.30 \\
\hline $\mathrm{TiO}_{2}$ & 1.04 & 0.85 & 0.48 & 0.78 & 0.72 & 0.58 & 0.75 & 1.07 & 0.94 & 0.96 \\
\hline $\mathrm{Al}_{2} \mathrm{O}_{3}$ & 2.64 & 2.46 & 1.66 & 2.82 & 3.26 & 3.82 & 2.59 & 2.90 & 2.60 & 2.67 \\
\hline $\mathrm{Cr}_{2} \mathrm{O}_{3}$ & 0.29 & 0.31 & 0.26 & 0.46 & 0.54 & 0.50 & 0.46 & 0.37 & 0.32 & 0.36 \\
\hline $\mathrm{FeO}$ & 3.60 & 3.38 & 3.12 & 3.40 & 3.85 & 3.94 & 3.78 & 4.21 & 4.09 & 3.87 \\
\hline $\mathrm{MnO}$ & 0.10 & 0.17 & 0.12 & 0.16 & 0.16 & 0.16 & 0.16 & 0.14 & 0.17 & 0.15 \\
\hline $\mathrm{MgO}$ & 16.52 & 16.51 & 16.93 & 16.53 & 16.93 & 18.71 & 16.67 & 15.93 & 16.37 & 16.10 \\
\hline $\mathrm{NiO}$ & 0.05 & 0.08 & 0.06 & 0.06 & 0.05 & 0.02 & 0.02 & 0.07 & 0.01 & 0.04 \\
\hline $\mathrm{CaO}$ & 23.51 & 23.85 & 24.17 & 22.93 & 22.25 & 19.64 & 22.44 & 22.75 & 22.91 & 23.60 \\
\hline $\mathrm{Na}_{2} \mathrm{O}$ & 0.37 & 0.36 & 0.26 & 0.42 & 0.42 & 0.43 & 0.37 & 0.39 & 0.46 & 0.41 \\
\hline $\mathrm{K}_{2} \mathrm{O}$ & - & - & - & 0.01 & 0.01 & 0.01 & - & 0.01 & 0.02 & 0.01 \\
\hline Total & 100.46 & 100.41 & 100.14 & 99.67 & 99.77 & 99.29 & 99.53 & 99.96 & 99.82 & 100.47 \\
\hline $\mathrm{Mg} \#$ & 89.1 & 89.7 & 90.6 & 89.6 & 88.7 & 89.4 & 88.7 & 87.1 & 87.7 & 88.1 \\
\hline Ca\# & 50.5 & 50.9 & 50.6 & 49.9 & 48.6 & 42.4 & 49.2 & 50.6 & 50.1 & 51.3 \\
\hline
\end{tabular}

Notes: Abbreviations as in Tables 1 and 4. Ca\# $=$ calcium number, $-=$ not detected. 
Table 10. Chemical composition of selected amphiboles.

\begin{tabular}{|c|c|c|c|c|c|c|c|c|c|}
\hline \multicolumn{10}{|c|}{ Edenitic/pargasitic amphiboles } \\
\hline \multirow{3}{*}{$\begin{array}{l}\text { Sample no.: } \\
\text { Rock name: }\end{array}$} & \multicolumn{6}{|c|}{110} & \multicolumn{3}{|c|}{125} \\
\hline & \multicolumn{6}{|c|}{$\mathrm{Gb}$} & \multicolumn{3}{|c|}{ OlGb } \\
\hline & \multirow[b]{2}{*}{6} & \multirow[b]{2}{*}{3} & \multirow[b]{2}{*}{2} & \multicolumn{3}{|c|}{ Profile } & \multirow[b]{2}{*}{3} & \multirow[b]{2}{*}{10} & \multirow[b]{2}{*}{6} \\
\hline$n:$ & & & & 2 & 3 & 1 & & & \\
\hline $\mathrm{SiO}_{2}$ & 45.27 & 45.95 & 48.52 & 48.74 & 46.63 & 49.21 & 43.90 & 47.42 & 42.36 \\
\hline $\mathrm{TiO}_{2}^{2}$ & 1.05 & 1.27 & 2.07 & 0.76 & 1.32 & 0.66 & 0.35 & 1.35 & 2.65 \\
\hline $\mathrm{Al}_{2} \mathrm{O}_{3}$ & 8.86 & 6.87 & 6.19 & 6.03 & 4.91 & 6.23 & 9.78 & 7.07 & 11.28 \\
\hline $\mathrm{Cr}_{2} \mathrm{O}_{3}$ & 0.03 & 0.05 & 0.12 & 0.04 & 0.13 & 0.12 & 0.02 & 0.01 & 0.02 \\
\hline $\mathrm{FeO}$ & 16.61 & 15.38 & 11.18 & 13.34 & 14.09 & 12.84 & 21.61 & 16.42 & 13.54 \\
\hline $\mathrm{MnO}$ & 0.25 & 0.24 & 0.20 & 0.22 & 0.21 & 0.23 & 0.38 & 0.30 & 0.22 \\
\hline $\mathrm{MgO}$ & 11.76 & 12.17 & 14.58 & 14.18 & 13.26 & 14.62 & 8.48 & 12.86 & 12.79 \\
\hline $\mathrm{NiO}$ & 0.08 & 0.07 & 0.02 & 0.09 & 0.04 & 0.02 & 0.03 & 0.04 & 0.06 \\
\hline $\mathrm{CaO}$ & 11.89 & 12.13 & 14.09 & 12.37 & 12.24 & 12.17 & 11.18 & 11.06 & 11.14 \\
\hline $\mathrm{Na}_{2} \mathrm{O}$ & 1.78 & 1.38 & 1.42 & 0.97 & 1.20 & 0.85 & 2.22 & 1.68 & 2.42 \\
\hline $\mathrm{K}_{2} \mathrm{O}$ & 0.39 & 0.32 & 0.01 & 0.22 & 0.22 & 0.17 & 0.12 & 0.21 & 0.38 \\
\hline $\mathrm{Cl}$ & 0.32 & 0.11 & 0.01 & 0.12 & 0.09 & 0.19 & 0.75 & 0.03 & 0.02 \\
\hline $\mathrm{H}_{2} \mathrm{O}$ & 1.96 & 1.97 & 2.04 & 1.99 & 1.98 & 1.97 & 1.80 & 2.07 & 2.03 \\
\hline Total & 100.25 & 95.91 & 100.44 & 97.95 & 97.64 & 97.42 & 100.62 & 100.52 & 98.91 \\
\hline $\mathrm{Mg \#}$ & 55.8 & 58.5 & 69.9 & 65.4 & 62.7 & 67.0 & 41.1 & 58.2 & 62.7 \\
\hline
\end{tabular}

Table 10 (continued).

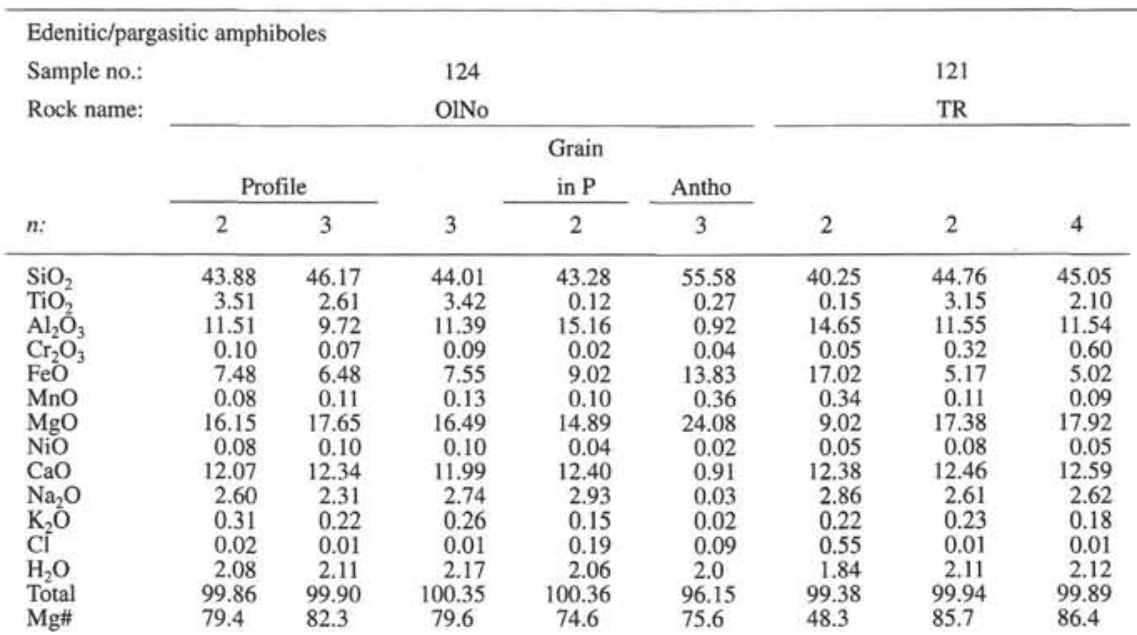

Table 10 (continued).

\begin{tabular}{|c|c|c|c|c|c|c|c|c|c|}
\hline \multicolumn{10}{|c|}{ Tremolitic/actinolitic amphiboles } \\
\hline Sample no.: & \multicolumn{3}{|c|}{125} & \multicolumn{4}{|c|}{123} & \multicolumn{2}{|c|}{124} \\
\hline \multirow[t]{2}{*}{ Rock name: } & \multicolumn{3}{|c|}{$\mathrm{OlGb}$} & \multirow{2}{*}{\multicolumn{3}{|c|}{ OINo }} & \multirow[b]{3}{*}{2} & \multicolumn{2}{|c|}{ OINo } \\
\hline & \multicolumn{3}{|c|}{ Profile } & & & & & \multirow[b]{2}{*}{2} & \multirow[b]{2}{*}{4} \\
\hline$n:$ & 3 & 2 & 4 & 2 & 5 & 2 & & & \\
\hline $\mathrm{SiO}_{2}$ & 48.15 & 43.01 & 49.16 & 48.80 & 50.08 & 51.30 & 48.04 & 56.84 & 56.78 \\
\hline $\mathrm{TiO}_{2}^{2}$ & 0.39 & 0.28 & 0.36 & 0.24 & 0.25 & 0.25 & 0.26 & 0.03 & - \\
\hline $\mathrm{Al}_{2} \mathrm{O}_{3}$ & 6.74 & 2.54 & 5.59 & 5.28 & 4.22 & 3.38 & 6.18 & 0.91 & 0.94 \\
\hline $\mathrm{Cr}_{2} \mathrm{O}_{3}$ & 0.03 & 0.02 & 0.02 & 0.01 & 0.04 & 0.02 & 0.01 & 0.02 & 0.02 \\
\hline $\mathrm{FeO}$ & 18.63 & 16.62 & 18.81 & 17.54 & 17.06 & 17.43 & 17.45 & 5.25 & 5.49 \\
\hline $\mathrm{MnO}$ & 0.44 & 0.50 & 0.48 & 0.37 & 0.38 & 0.50 & 0.39 & 0.22 & 0.22 \\
\hline $\mathrm{MgO}$ & 11.51 & 14.21 & 11.84 & 12.61 & 13.50 & 14.25 & 12.43 & 21.60 & 21.46 \\
\hline $\mathrm{NiO}$ & 0.03 & 0.05 & 0.03 & 0.02 & 0.05 & 0.02 & 0.04 & 0.07 & 0.06 \\
\hline $\mathrm{CaO}$ & 10.45 & 10.85 & 9.87 & 10.80 & 10.81 & 9.79 & 10.85 & 12.59 & 12.33 \\
\hline $\mathrm{Na}_{2} \mathrm{O}$ & 1.59 & 0.65 & 1.33 & 1.28 & 1.10 & 0.78 & 1.49 & 0.35 & 0.32 \\
\hline $\mathrm{K}_{2} \mathrm{O}$ & 0.07 & 0.04 & 0.08 & 0.13 & 0.07 & 0.06 & 0.13 & 0.01 & - \\
\hline $\mathrm{Cl}$ & 0.20 & 0.06 & 0.08 & 0.17 & 0.12 & 0.09 & 0.20 & 0.02 & 0.01 \\
\hline $\mathrm{H}_{2} \mathrm{O}$ & 2.01 & 2.10 & 2.05 & 2.00 & 2.05 & 2.09 & 2.01 & 2.18 & 2.18 \\
\hline Total & 100.24 & 100.93 & 99.70 & 99.25 & 99.71 & 99.66 & 99.48 & 100.09 & 99.81 \\
\hline Mg\# & 52.4 & 60.4 & 52.8 & 56.0 & 58.5 & 59.3 & 55.9 & 88.0 & 87.4 \\
\hline
\end{tabular}


Table 10 (continued).

\begin{tabular}{|c|c|c|c|c|}
\hline \multicolumn{5}{|c|}{ Tremolitic/actinolitic amphiboles } \\
\hline Sample no: & & 121 & & \multirow[b]{3}{*}{$\begin{array}{c}\text { Grain in } \\
\text { clino- } \\
\text { pyroxene }\end{array}$} \\
\hline Rock name: & & TR & & \\
\hline \multirow[b]{2}{*}{$n:$} & \multicolumn{3}{|c|}{ Profile } & \\
\hline & 2 & 2 & 1 & 3 \\
\hline $\mathrm{SiO}_{2}$ & 56.38 & 58.26 & 56.19 & 55.04 \\
\hline $\mathrm{TiO}_{2}$ & 0.02 & 0.01 & 0.05 & 0.14 \\
\hline $\mathrm{Al}_{2} \mathrm{O}_{3}$ & 1.70 & 0.18 & 1.19 & 2.41 \\
\hline $\mathrm{Cr}_{2} \mathrm{O}_{3}$ & 0.03 & 0.02 & - & 0.18 \\
\hline $\mathrm{FeO}$ & 3.93 & 2.65 & 5.20 & 4.60 \\
\hline $\mathrm{MnO}$ & 0.11 & 0.13 & 0.13 & 0.21 \\
\hline $\mathrm{MgO}$ & 21.72 & 22.84 & 21.67 & 21.37 \\
\hline $\mathrm{NiO}$ & 0.09 & 0.04 & 0.01 & 0.07 \\
\hline $\mathrm{CaO}$ & 13.55 & 13.80 & 12.80 & 12.93 \\
\hline $\mathrm{Na}_{2} \mathrm{O}$ & 0.56 & 0.07 & 0.43 & 0.70 \\
\hline $\mathrm{K}_{2} \mathrm{O}$ & 0.02 & - & 0.02 & 0.02 \\
\hline $\mathrm{Cl}^{2}$ & 0.03 & 0.01 & 0.02 & 0.03 \\
\hline $\mathrm{H}_{2} \mathrm{O}$ & 2.16 & 2.19 & 2.17 & 2.16 \\
\hline Total & 100.30 & 100.20 & 99.88 & 99.86 \\
\hline Mg\# & 90.8 & 93.9 & 88.1 & 89.2 \\
\hline
\end{tabular}

Notes: Abbreviations as in Tables 1 and 4. Cpx = clinopyroxene, in $\mathrm{P}=$ plagioclase, Antho $=$ anthophyllite; $-=$ not detected.

Table 11. Chemical composition of selected plagioclases.

\begin{tabular}{|c|c|c|c|c|c|c|c|c|c|c|c|c|}
\hline \multirow{3}{*}{$\begin{array}{l}\text { Sample no:: } \\
\text { Rock name: }\end{array}$} & \multicolumn{6}{|c|}{102} & \multicolumn{6}{|c|}{110} \\
\hline & \multicolumn{6}{|c|}{$\mathrm{Gb}$} & \multicolumn{6}{|c|}{$\mathrm{Gb}$} \\
\hline & \multicolumn{6}{|c|}{ Profile 1} & \multicolumn{6}{|c|}{ Profile 2} \\
\hline$n:$ & 1 & 2 & 4 & 2 & 4 & 4 & 10 & 4 & 4 & 2 & 2 & 1 \\
\hline $\mathrm{SiO}_{2}$ & 54.13 & 53.66 & 54.24 & 55.02 & 54.96 & 55.25 & 52.71 & 53.82 & 54.55 & 55.89 & 54.57 & 53.18 \\
\hline $\mathrm{TiO}_{2}$ & 0.10 & 0.05 & 0.08 & 0.06 & 0.04 & 0.04 & 0.02 & 0.03 & 0.02 & 0.04 & 0.02 & 0.04 \\
\hline $\mathrm{Al}_{2} \mathrm{O}_{3}$ & 28.42 & 28.22 & 28.39 & 27.86 & 27.98 & 27.71 & 30.07 & 29.40 & 28.89 & 27.93 & 28.70 & 29.79 \\
\hline $\mathrm{Fe}_{2} \mathrm{O}_{3}$ & 0.28 & 0.21 & 0.27 & 0.30 & 0.26 & 0.28 & 0.11 & 0.05 & 0.06 & 0.06 & 0.17 & 0.11 \\
\hline $\mathrm{MgO}$ & 0.03 & 0.01 & 0.03 & 0.02 & 0.03 & 0.03 & 0.02 & 0.02 & 0.01 & 0.03 & 0.02 & 0.03 \\
\hline $\mathrm{CaO}$ & 11.31 & 12.18 & 11.25 & 10.48 & 10.74 & 10.39 & 12.81 & 11.98 & 11.44 & 10.33 & 10.98 & 12.28 \\
\hline $\mathrm{Na}_{2} \mathrm{O}$ & 5.53 & 5.52 & 5.56 & 6.00 & 5.93 & 6.10 & 4.62 & 5.15 & 5.47 & 6.16 & 5.58 & 4.89 \\
\hline $\mathrm{K}_{2} \mathrm{O}$ & 0.05 & 0.09 & 0.07 & 0.08 & 0.07 & 0.06 & 0.07 & 0.06 & 0.07 & 0.11 & 0.09 & 0.07 \\
\hline Total & 99.85 & 99.94 & 99.89 & 99.82 & 100.01 & 99.86 & 100.43 & 100.51 & 100.51 & 100.57 & 100.13 & 100.38 \\
\hline An\# & 52.9 & 54.7 & 52.5 & 49.0 & 49.8 & 48.4 & 60.3 & 56.0 & 53.4 & 47.9 & 51.9 & 57.9 \\
\hline
\end{tabular}

Table 11 (continued).

\begin{tabular}{|c|c|c|c|c|c|c|c|c|}
\hline \multirow{4}{*}{$\begin{array}{l}\text { Sample no: } \\
\text { Rock name: } \\
n:\end{array}$} & \multicolumn{8}{|c|}{118} \\
\hline & \multirow{2}{*}{\multicolumn{6}{|c|}{$\begin{array}{c}\mathrm{Gb} \\
\text { Profile } 3\end{array}$}} & \multirow{2}{*}{\multicolumn{2}{|c|}{$\begin{array}{l}\text { At contact with } \\
\mathrm{cpx} \Rightarrow \text { amph }\end{array}$}} \\
\hline & & & & & & & & \\
\hline & 2 & 3 & 2 & 2 & 3 & 1 & 1 & 1 \\
\hline $\mathrm{SiO}_{2}$ & 52.79 & 52.79 & 52.91 & 53.51 & 53.15 & 51.84 & 49.88 & 51.76 \\
\hline $\mathrm{TiO}_{2}$ & 0.06 & 0.10 & 0.10 & 0.10 & 0.08 & 0.05 & 0.07 & 0.04 \\
\hline $\mathrm{Al}_{2} \mathrm{O}_{3}$ & 29.30 & 29.35 & 28.90 & 28.72 & 29.01 & 30.36 & 29.71 & 29.67 \\
\hline $\mathrm{Fe}_{2} \mathrm{O}_{3}$ & 0.28 & 0.24 & 0.25 & 0.24 & 0.24 & 0.18 & 0.92 & 0.58 \\
\hline $\mathrm{MgO}$ & 0.03 & 0.03 & 0.05 & 0.02 & 0.02 & 0.01 & 0.90 & 0.32 \\
\hline $\mathrm{CaO}$ & 12.16 & 12.39 & 12.03 & 11.63 & 11.82 & 13.16 & 13.19 & 13.04 \\
\hline $\mathrm{Na}_{2} \mathrm{O}$ & 5.06 & 4.92 & 5.10 & 5.34 & 5.30 & 4.50 & 3.89 & 4.36 \\
\hline $\mathrm{K}_{2} \mathrm{O}$ & 0.06 & 0.06 & 0.06 & 0.07 & 0.07 & 0.05 & 0.04 & 0.07 \\
\hline Total & 99.84 & 99.86 & 99.40 & 99.63 & 99.69 & 100.15 & 98.60 & 99.84 \\
\hline An\# & 57.1 & 58.0 & 56.5 & 54.4 & 55.0 & 61.6 & 65.1 & 62.1 \\
\hline
\end{tabular}


Table 11 (continued).

\begin{tabular}{|c|c|c|c|c|c|c|c|c|c|}
\hline \multirow{4}{*}{$\begin{array}{l}\text { Sample no:: } \\
\text { Rock name: } \\
n:\end{array}$} & \multicolumn{9}{|c|}{104} \\
\hline & \multicolumn{9}{|c|}{ OIGb } \\
\hline & \multicolumn{8}{|c|}{ Profile 1} & \multirow{2}{*}{$\begin{array}{c}\begin{array}{c}\text { Grain } \\
\text { in amph }\end{array} \\
2\end{array}$} \\
\hline & 2 & 2 & 3 & 2 & 5 & 4 & 1 & 1 & \\
\hline $\mathrm{SiO}_{2}$ & 51.32 & 49.96 & 50.23 & 50.74 & 50.02 & 51.23 & 49.38 & 51.46 & 59.76 \\
\hline $\mathrm{TiO}_{2}^{2}$ & 0.03 & 0.06 & 0.06 & 0.04 & 0.05 & 0.06 & 0.04 & 0.08 & 0.06 \\
\hline $\mathrm{Al}_{2} \mathrm{O}_{3}$ & 30.32 & 31.02 & 30.87 & 30.51 & 30.76 & 30.11 & 31.39 & 30.32 & 25.32 \\
\hline $\mathrm{Fe}_{2} \mathrm{O}_{3}$ & 0.16 & 0.20 & 0.18 & 0.20 & 0.24 & 0.15 & 0.19 & 0.22 & 0.19 \\
\hline $\mathrm{MgO}$ & 0.03 & 0.02 & 0.03 & 0.02 & 0.03 & 0.02 & 0.01 & 0.02 & 0.02 \\
\hline $\mathrm{CaO}$ & 13.71 & 14.68 & 14.34 & 13.72 & 14.32 & 13.38 & 14.72 & 13.54 & 7.21 \\
\hline $\mathrm{Na}_{2} \mathrm{O}$ & 4.29 & 3.73 & 3.88 & 4.08 & 3.85 & 4.38 & 3.53 & 4.37 & 7.50 \\
\hline $\mathrm{K}_{2} \mathrm{O}$ & 0.05 & 0.04 & 0.06 & 0.04 & 0.04 & 0.06 & 0.03 & 0.05 & 0.18 \\
\hline Total & 99.91 & 99.71 & 99.65 & 99.35 & 99.31 & 99.33 & 99.29 & 100.06 & 100.24 \\
\hline An\# & 63.7 & 68.3 & 66.9 & 64.9 & 67.2 & 62.6 & 69.6 & 63.0 & 34.3 \\
\hline
\end{tabular}

Table 11 (continued).

\begin{tabular}{|c|c|c|c|c|c|c|c|c|}
\hline \multirow{4}{*}{$\begin{array}{l}\text { Sample no: } \\
\text { Rock name: } \\
n:\end{array}$} & \multicolumn{8}{|c|}{119} \\
\hline & \multicolumn{8}{|c|}{$\mathrm{OIGb}$} \\
\hline & \multicolumn{5}{|c|}{ Profile 3} & \multicolumn{3}{|c|}{ Profile 4} \\
\hline & 1 & 1 & 1 & 1 & 1 & 2 & 1 & 1 \\
\hline $\mathrm{SiO}_{2}$ & 49.93 & 50.68 & 50.79 & 50.49 & 50.33 & 48.94 & 52.06 & 50.23 \\
\hline $\mathrm{TiO}_{2}$ & - & 0.02 & 0.02 & 0.03 & 0.01 & 0.05 & 0.06 & 0.04 \\
\hline $\mathrm{Al}_{2} \mathrm{O}_{3}$ & 31.56 & 31.27 & 31.04 & 31.24 & 31.39 & 32.22 & 30.18 & 31.18 \\
\hline $\mathrm{Fe}_{2} \mathrm{O}_{3}$ & 0.15 & 0.16 & 0.14 & 0.19 & 0.14 & 0.28 & 0.26 & 0.26 \\
\hline $\mathrm{MgO}^{3}$ & 0.01 & 0.02 & 0.01 & 0.02 & 0.02 & 0.03 & 0.01 & 0.05 \\
\hline $\mathrm{CaO}$ & 15.04 & 14.64 & 14.37 & 14.28 & 14.88 & 15.88 & 13.10 & 14.49 \\
\hline $\mathrm{Na}_{2} \mathrm{O}$ & 3.52 & 3.63 & 3.81 & 3.70 & 3.51 & 2.82 & 4.57 & 3.68 \\
\hline $\mathrm{K}_{2} \mathrm{O}$ & 0.05 & 0.01 & 0.03 & 0.03 & 0.01 & 0.01 & 0.03 & 0.04 \\
\hline Total & 100.26 & 100.43 & 100.21 & 99.98 & 100.29 & 100.23 & 100.27 & 99.97 \\
\hline An\# & 70.0 & 69.0 & 67.4 & 68.0 & 70.0 & 75.6 & 61.2 & 68.4 \\
\hline
\end{tabular}

Table 11 (continued).

\begin{tabular}{|c|c|c|c|c|c|c|c|c|c|c|}
\hline \multirow{4}{*}{$\begin{array}{l}\text { Sample no.: } \\
\text { Rock name: } \\
n:\end{array}$} & \multicolumn{10}{|c|}{125} \\
\hline & \multicolumn{10}{|c|}{$\mathrm{OlGb}$} \\
\hline & \multicolumn{10}{|c|}{ Profile 5} \\
\hline & 10 & 8 & 6 & 5 & 4 & 3 & 2 & 3 & 6 & 2 \\
\hline $\mathrm{SiO}_{2}$ & 50.67 & 50.33 & 50.07 & 49.17 & 48.50 & 48.16 & 49.11 & 49.81 & 50.22 & 50.39 \\
\hline $\mathrm{TiO}_{2}$ & 0.05 & 0.06 & 0.03 & 0.02 & 0.04 & 0.02 & 0.03 & 0.05 & 0.05 & 0.03 \\
\hline $\mathrm{Al}_{2} \mathrm{O}_{3}$ & 31.06 & 31.62 & 32.08 & 32.57 & 33.08 & 32.97 & 32.52 & 31.81 & 31.84 & 31.78 \\
\hline $\mathrm{Fe}_{2} \mathrm{O}_{3}$ & 0.17 & 0.11 & 0.07 & 0.06 & 0.08 & 0.10 & 0.14 & 0.13 & 0.12 & 0.07 \\
\hline $\mathrm{MgO}$ & 0.03 & 0.03 & 0.01 & 0.03 & 0.03 & 0.02 & 0.01 & 0.02 & 0.02 & 0.01 \\
\hline $\mathrm{CaO}$ & 14.15 & 14.71 & 14.97 & 15.69 & 16.28 & 16.45 & 15.67 & 14.87 & 14.82 & 14.73 \\
\hline $\mathrm{Na}_{2} \mathrm{O}$ & 3.76 & 3.54 & 3.34 & 2.97 & $\begin{array}{r}10.20 \\
2.54\end{array}$ & 2.40 & 2.90 & 3.30 & 3.39 & 3.53 \\
\hline $\mathrm{K}, \mathrm{O}$ & 0.06 & 0.05 & 0.02 & 0.03 & 0.02 & 0.03 & 0.03 & 0.02 & 0.04 & 0.05 \\
\hline Total & 99.95 & 100.45 & 100.59 & 100.54 & 100.57 & 100.15 & 100.41 & 100.01 & 100.50 & 100.59 \\
\hline An\# & 67.3 & 69.5 & 71.2 & 74.4 & 77.9 & 79.1 & 74.8 & 71.3 & 70.6 & 69.5 \\
\hline
\end{tabular}

Table 11 (continued).

\begin{tabular}{|c|c|c|c|c|c|c|c|c|c|c|c|}
\hline Sample no.: & \multicolumn{11}{|c|}{113} \\
\hline \multirow[t]{2}{*}{ Rock name: } & \multicolumn{11}{|c|}{ GbNo } \\
\hline & \multicolumn{11}{|c|}{ Profile 2} \\
\hline$n:$ & 3 & 4 & 3 & 4 & 3 & 2 & 2 & 5 & 3 & 7 & 9 \\
\hline $\mathrm{SiO}_{2}$ & 53.41 & 52.01 & 51.74 & 52.00 & 51.86 & 52.01 & 52.49 & 52.63 & 52.79 & 52.91 & 52.68 \\
\hline $\mathrm{TiO}_{2}$ & 0.03 & 0.05 & 0.06 & 0.05 & 0.06 & 0.05 & 0.07 & 0.07 & 0.05 & 0.06 & 0.06 \\
\hline $\mathrm{Al}_{2} \mathrm{O}_{3}$ & 29.61 & 30.54 & 30.55 & 30.50 & 30.47 & 30.28 & 30.12 & 30.03 & 30.02 & 29.94 & 30.04 \\
\hline $\mathrm{Fe}_{2} \mathrm{O}_{3}$ & 0.09 & 0.13 & 0.18 & 0.16 & 0.25 & 0.28 & 0.28 & 0.21 & 0.15 & 0.16 & 0.21 \\
\hline $\mathrm{MgO}$ & 0.01 & 0.02 & 0.03 & 0.03 & 0.04 & 0.03 & 0.02 & 0.03 & 0.02 & 0.03 & 0.02 \\
\hline $\mathrm{CaO}$ & 12.22 & 13.33 & 13.55 & 13.39 & 13.50 & 13.27 & 12.98 & 12.89 & 12.82 & 12.73 & 12.86 \\
\hline $\mathrm{Na}_{2} \mathrm{O}$ & 5.04 & 4.34 & 4.28 & 4.39 & 4.28 & 4.44 & 4.56 & 4.61 & 4.70 & 4.67 & 4.66 \\
\hline $\mathrm{K}_{2} \mathrm{O}$ & 0.05 & 0.05 & 0.03 & 0.04 & 0.05 & 0.05 & 0.04 & 0.05 & 0.05 & 0.05 & 0.05 \\
\hline Total & 100.46 & 100.47 & 100.42 & 100.56 & 100.51 & 100.41 & 100.56 & 100.52 & 100.60 & 100.55 & 100.58 \\
\hline An\# & 57.1 & 62.8 & 63.5 & 62.7 & 63.3 & 62.1 & 61.0 & 60.5 & 60.0 & 59.9 & 60.2 \\
\hline
\end{tabular}


Table 11 (continued).

\begin{tabular}{|c|c|c|c|c|c|c|c|c|c|c|}
\hline Sample no.: & \multicolumn{4}{|c|}{123} & \multicolumn{6}{|c|}{124} \\
\hline \multirow[t]{3}{*}{ Rock name: } & \multicolumn{4}{|c|}{ OINo } & \multicolumn{6}{|c|}{ OINo } \\
\hline & \multicolumn{4}{|c|}{ Grains } & \multicolumn{6}{|c|}{ Profile 1} \\
\hline & 2 & 3 & 3 & 3 & 4 & 5 & 2 & 2 & 3 & 2 \\
\hline $\mathrm{SiO}_{2}$ & 57.73 & 57.41 & 56.67 & 56.18 & 48.49 & 48.55 & 49.53 & 49.04 & 49.86 & 49.27 \\
\hline $\mathrm{TiO}_{2}$ & 0.02 & 0.02 & 0.03 & 0.03 & 0.04 & 0.03 & 0.02 & 0.04 & 0.02 & 0.02 \\
\hline $\mathrm{Al}_{2} \mathrm{O}_{3}$ & 26.63 & 27.10 & 27.45 & 27.69 & 33.04 & 33.04 & 32.52 & 32.55 & 32.13 & 32.73 \\
\hline $\mathrm{Fe}_{2} \mathrm{O}_{3}$ & 0.13 & 0.12 & 0.10 & 0.09 & 0.09 & 0.06 & 0.04 & 0.09 & 0.06 & 0.10 \\
\hline $\mathrm{MgO}^{3}$ & 0.02 & 0.02 & 0.03 & 0.02 & 0.03 & - & 0.02 & 0.02 & 0.01 & 0.01 \\
\hline $\mathrm{CaO}$ & 8.64 & 9.16 & 9.68 & 10.11 & 16.22 & 16.16 & 15.38 & 15.73 & 15.16 & 15.48 \\
\hline $\mathrm{Na}_{2} \mathrm{O}$ & 7.19 & 6.69 & 6.51 & 6.25 & 2.64 & 2.73 & 3.13 & 2.90 & 3.29 & 2.98 \\
\hline $\mathrm{K}_{2} \mathrm{O}$ & 0.05 & 0.10 & 0.11 & 0.10 & 0.03 & 0.02 & 0.04 & 0.03 & 0.03 & 0.03 \\
\hline Total & 100.41 & 100.62 & 100.58 & 100.47 & 100.58 & 100.61 & 100.66 & 100.40 & 100.57 & 100.62 \\
\hline An\# & 39.9 & 42.9 & 44.8 & 46.9 & 77.0 & 76.5 & 72.9 & 74.9 & 71.6 & 74.1 \\
\hline
\end{tabular}

Table 11 (continued).

\begin{tabular}{|c|c|c|c|c|c|c|c|c|c|c|}
\hline Sample no.: & \multicolumn{10}{|c|}{121} \\
\hline \multirow[t]{3}{*}{ Rock name: } & \multicolumn{10}{|c|}{ TR } \\
\hline & \multicolumn{10}{|c|}{ Profile 1} \\
\hline & 1 & 1 & 1 & 5 & 1 & 1 & 2 & 1 & 1 & 2 \\
\hline $\mathrm{SiO}_{2}$ & 46.81 & 48.62 & 45.22 & 48.49 & 47.77 & 47.45 & 47.96 & 46.39 & 47.66 & 48.07 \\
\hline $\mathrm{TiO}_{2}$ & 0.03 & 0.02 & - & 0.05 & 0.02 & 0.02 & 0.03 & 0.02 & 0.02 & 0.03 \\
\hline $\mathrm{Al}_{2} \mathrm{O}_{3}$ & 34.15 & 32.07 & 35.37 & 32.95 & 33.41 & 33.84 & 33.26 & 34.46 & 33.55 & 33.37 \\
\hline $\mathrm{Fe}_{2} \mathrm{O}_{3}$ & 0.25 & 0.33 & 0.25 & 0.14 & 0.15 & 0.09 & 0.12 & 0.10 & 0.08 & 0.07 \\
\hline $\mathrm{MgO}^{3}$ & 0.02 & 0.06 & 0.02 & 0.02 & 0.02 & 0.02 & 0.01 & 0.02 & 0.02 & 0.01 \\
\hline $\mathrm{CaO}$ & 17.59 & 15.97 & 18.90 & 16.17 & 16.90 & 17.14 & 16.49 & 17.94 & 16.97 & 16.58 \\
\hline $\mathrm{Na}_{2} \mathrm{O}$ & 1.96 & 2.45 & 1.09 & 2.82 & 2.38 & 2.21 & 2.56 & 1.74 & 2.31 & 2.57 \\
\hline $\mathrm{K}_{2} \mathrm{O}$ & 0.01 & - & - & 0.02 & 0.01 & 0.02 & 0.02 & - & 0.02 & 0.02 \\
\hline Total & 100.82 & 99.52 & 100.85 & 100.66 & 100.66 & 100.79 & 100.45 & 100.67 & 100.63 & 100.72 \\
\hline An\# & 83.2 & 78.3 & 90.5 & 76.0 & 79.6 & 81.0 & 78.0 & 85.1 & 80.1 & 78.1 \\
\hline
\end{tabular}

Table 11 (continued).

\begin{tabular}{|c|c|c|c|c|c|c|c|c|c|c|}
\hline \multirow{4}{*}{$\begin{array}{l}\text { Sample no.: } \\
\text { Rock name: } \\
n:\end{array}$} & \multicolumn{10}{|c|}{121} \\
\hline & \multicolumn{10}{|c|}{ TR } \\
\hline & \multicolumn{5}{|c|}{ Profile 2} & \multicolumn{5}{|c|}{ Profile 3} \\
\hline & 2 & 1 & 2 & 2 & 2 & 1 & 2 & 1 & 1 & 2 \\
\hline $\mathrm{SiO}_{2}$ & 48.77 & 48.50 & 48.40 & 48.44 & 48.90 & 48.52 & 48.91 & 49.35 & 48.64 & 48.77 \\
\hline $\mathrm{TiO}_{2}$ & 0.06 & 0.08 & 0.08 & 0.12 & 0.08 & 0.06 & 0.09 & 0.07 & 0.04 & 0.05 \\
\hline $\mathrm{Al}_{2} \mathrm{O}_{3}$ & 32.67 & 33.06 & 32.92 & 32.69 & 32.64 & 32.73 & 32.62 & 31.95 & 32.72 & 32.74 \\
\hline $\mathrm{Fe}_{2} \mathrm{O}_{3}$ & 0.26 & 0.19 & 0.26 & 0.23 & 0.22 & 0.13 & 0.19 & 0.22 & 0.18 & 0.18 \\
\hline $\mathrm{MgO}$ & 0.06 & 0.06 & 0.04 & 0.03 & 0.04 & 0.02 & 0.04 & 0.06 & 0.04 & 0.03 \\
\hline $\mathrm{CaO}$ & 15.86 & 16.17 & 16.24 & 16.03 & 15.86 & 16.18 & 15.92 & 15.09 & 15.88 & 15.97 \\
\hline $\mathrm{Na}_{2} \mathrm{O}$ & 2.87 & 2.80 & 2.66 & 2.80 & 2.84 & 2.81 & 2.98 & 3.26 & 3.01 & 2.89 \\
\hline $\mathrm{K}_{2} \mathrm{O}$ & 0.03 & - & 0.02 & 0.03 & 0.03 & 0.03 & 0.03 & 0.03 & 0.01 & 0.02 \\
\hline Total & 100.58 & 100.86 & 100.62 & 100.37 & 100.61 & 100.48 & 100.78 & 100.03 & 100.52 & 100.65 \\
\hline An\# & 75.2 & 76.1 & 77.1 & 75.8 & 75.4 & 76.0 & 74.6 & 71.8 & 74.4 & 75.3 \\
\hline
\end{tabular}

Table 11 (continued).

\begin{tabular}{|c|c|c|c|c|c|c|c|c|c|c|}
\hline \multirow{4}{*}{$\begin{array}{l}\text { Sample no.: } \\
\text { Rock name: } \\
n:\end{array}$} & \multicolumn{10}{|c|}{122} \\
\hline & \multicolumn{10}{|c|}{ TR } \\
\hline & \multicolumn{10}{|c|}{ Profile } \\
\hline & 3 & 1 & 1 & 1 & 1 & 1 & 2 & 1 & 1 & 1 \\
\hline $\mathrm{SiO}_{2}$ & 48.88 & 52.19 & 50.95 & 52.70 & 49.48 & 51.32 & 49.80 & 51.72 & 49.93 & 49.28 \\
\hline $\mathrm{TiO}_{2}$ & 0.03 & 0.02 & 0.03 & 0.02 & 0.02 & 0.03 & 0.02 & 0.02 & 0.03 & 0.02 \\
\hline $\mathrm{Al}_{2} \hat{\mathrm{O}}_{3}$ & 32.61 & 30.65 & 31.17 & 30.42 & 32.46 & 31.35 & 32.21 & 30.79 & 31.79 & 32.41 \\
\hline $\mathrm{Fe}_{2} \mathrm{O}_{3}$ & 0.13 & 0.05 & 0.12 & 0.14 & 0.06 & 0.13 & 0.07 & 0.05 & 0.08 & 0.06 \\
\hline $\mathrm{MgO}^{3}$ & 0.03 & 0.01 & 0.02 & 0.02 & 0.01 & 0.02 & 0.02 & 0.02 & 0.02 & 0.01 \\
\hline $\mathrm{CaO}$ & 15.81 & 13.28 & 14.28 & 12.69 & 15.45 & 13.84 & 15.08 & 13.67 & 14.83 & 15.25 \\
\hline $\mathrm{Na}_{2} \mathrm{O}$ & 2.88 & 4.54 & 3.96 & 4.68 & 3.19 & 3.82 & 3.34 & 4.26 & 3.41 & 3.18 \\
\hline $\mathrm{K}_{2} \mathrm{O}$ & 0.03 & 0.10 & 0.04 & 0.07 & 0.01 & 0.06 & 0.01 & 0.06 & 0.04 & 0.04 \\
\hline Total & 100.40 & 100.84 & 100.57 & 100.74 & 100.68 & 100.57 & 100.55 & 100.59 & 100.13 & 100.25 \\
\hline An\# & 75.1 & 61.6 & 66.5 & 59.7 & 72.8 & 66.5 & 71.4 & 63.8 & 70.5 & 72.4 \\
\hline
\end{tabular}

Notes: Abbreviations as in Tables 1 and $4 ; \mathrm{amph}=\mathrm{amphibole,} \mathrm{cpx}=$ clinopyroxene; An\# is defined in the text; $-=$ not detected. 